\title{
Transsynaptic interactions between IgSF proteins DIP- $\alpha$ and Dpr10 are required for motor neuron targeting specificity in Drosophila
}

\author{
James Ashley ${ }^{1}$, Violet Sorrentino ${ }^{1}$, Sonal Nagarkar-Jaiswal2 ${ }^{2}$, Liming Tan ${ }^{3}$, Shuwa Xu3 , Qi \\ $\mathrm{Xiao}^{3}$, Kai Zinn ${ }^{4 *}$, Robert A. Carrillo ${ }^{1^{*}}$ \\ ${ }^{1}$ Department of Molecular Genetics and Cell Biology, University of Chicago, Chicago, IL \\ 60637, USA \\ 2Department of Molecular and Human Genetics, Program in Developmental Biology, \\ Howard Hughes Medical Institute, Jan and Dan Neurological Research Institute at TCH, \\ Baylor College of Medicine, Houston, TX 77030, USA \\ ${ }^{3}$ Department of Biological Chemistry, Howard Hughes Medical Institute, David Geffen \\ School of Medicine, University of California, Los Angeles, Los Angeles, CA 90095, USA \\ ${ }^{4}$ Division of Biology and Biological Engineering, California Institute of Technology, \\ Pasadena, CA 91125, USA \\ *Correspondence: robertcarrillo@uchicago.edu (R.A.C.), zinnk@caltech.edu (K.Z.)
}

\begin{abstract}
The Drosophila larval neuromuscular system provides an ideal context in which to study synaptic partner choice, because it contains a small number of pre- and postsynaptic cells connected in an invariant pattern. The discovery of interactions between two subfamilies of IgSF cell surface proteins, the Dprs and the DIPs, provided new candidates for cellular labels controlling synaptic specificity. Here we show that DIP- $\alpha$ is expressed by two identified motor neurons, while its binding partner Dpr10 is expressed by postsynaptic muscle targets. Removal of either DIP- $\alpha$ or Dpr10 results in loss of specific axonal branches and NMJs formed by one motor neuron, MNISN-1s, while other branches of the MNISN-1s axon develop normally. The temporal and spatial expression pattern of dpr10 correlates with muscle innervation by MNISN-1s during embryonic development. We propose a model whereby DIP- $\alpha$ and Dpr10 on opposing synaptic partners interact with each other to generate proper motor neuron connectivity.
\end{abstract}




\section{INTRODUCTION}

The proper wiring of neural circuits is essential for animal behavior, and alterations in connectivity are linked to neurological disease phenotypes in humans (Rowe, 2010). Thus, identifying cell-surface molecules involved in neural wiring is critical for understanding biological mechanisms in normal development and in diseased states. Using genetics to uncover these mechanisms has been difficult, partially due to the fact that achieving the necessary precision appears to require partially redundant biochemical interactions.

One of the simplest and most accessible systems in which to study the genetic determination of synaptic connectivity patterns is the Drosophila larval neuromuscular system. In each larval abdominal hemisegment, 35 identified motor neurons innervate a set of 30 muscle fibers. Each motor neuron chooses one or more specific muscle fibers as synaptic targets, and the map of connections is almost invariant. Drosophila neuromuscular junction (NMJ) synapses are glutamatergic and use orthologs of mammalian AMPA receptors for synaptic transmission. Many scaffolding and regulatory proteins that modulate these receptors are conserved between insects and vertebrates. The sizes and strengths of Drosophila NMJs are regulated by retrograde signaling from their postsynaptic muscle targets. In addition to this developmental plasticity, NMJ synapses also exhibit short-term and homeostatic plasticity. These features make the Drosophila NMJ a useful genetic model system for excitatory glutamatergic synapses in the mammalian brain (Broadie and Bate, 1993; Keshishian et al., 1996; Menon et al., 2013).

Although many molecules involved in axon guidance, NMJ morphology, and synaptic activity have been identified through genetic and reverse genetic experiments, we still lack an understanding of the mechanisms by which individual larval muscle fibers are recognized as synaptic targets by Drosophila motor axons. Gain-of-function (GOF) experiments suggest that individual muscles are labeled by cell-surface proteins (CSPs) that can define them as targets for motor axons. 30 CSPs have been identified that cause motor axons to mistarget when they are ubiquitously expressed in muscles. These proteins contain a variety of extracellular domain (XCD) types, including immunoglobulin superfamily (IgSF) domains and leucine-rich repeat (LRR) sequences. Some of these proteins are normally expressed on subsets of muscles in embryos, suggesting that they could act as molecular signatures during motor axon targeting (Kurusu et al., 2008). However, none of the CSPs identified thus far are required for innervation of the muscles that express them, suggesting that they have partially redundant functions (Chiba et al., 1995; Inaki et al., 2007; Kurusu et al., 2008; Taniguchi et al., 2000; Winberg et al., 1998). In loss-of-function (LOF) mutants lacking CSPs expressed on muscle fibers or the receptors for these proteins on motor axons, innervation occurs normally in most cases. To our knowledge, there are no published LOF mutations in CSP genes that cause high-penetrance failures of innervation of specific muscle fibers.

A network of new candidates for synaptic targeting molecules was recently identified through a global in vitro 'interactome' screen (Ozkan et al., 2013). In this network, the 'Dprome', a set of 21 proteins with two IgSF domains, the Dprs, interact in a complex pattern with a set of 9 proteins with three IgSF domains, called DIPs. We and others have examined 
the expression patterns of many Dprs and DIPs, and found that each is expressed in a small and unique subset of neurons in the larval ventral nerve cord and pupal brain (Carrillo et al., 2015; Tan et al., 2015).

We studied the functions of one Dpr-DIP binding pair, Dpr11-DIP- $\gamma$, in both the larval neuromuscular system and the pupal optic lobe. Loss of either $d p r 11$ or DIP- $\gamma$ produced phenotypes affecting NMJ morphology and retrograde bone morphogenetic protein (BMP) signaling, but did not alter NMJ connectivity patterns. DIP- $\gamma$ is expressed in most motor neurons, so it is unlikely to be involved in recognition of targets by specific motor neurons. In the optic lobe, however, DIP- $\gamma$ is selectively expressed in amacrine neurons that are postsynaptic to photoreceptor neurons that express Dpr11, suggesting that Dpr11-DIP- $\gamma$ interactions may be important in determining synaptic connectivity patterns (Carrillo et al., 2015). For several other Dpr-DIP in vitro binding pairs, optic lobe neurons expressing a Dpr are also synaptically connected to neurons expressing the cognate DIP (Tan et al., 2015).

Based on these findings, we surveyed DIP expression in the larval neuromuscular system, in order to identify DIPs whose expression is restricted to subsets of motor neurons. Remarkably, DIP- $\alpha$ is expressed by only two motor neurons in each hemisegment. There are two types of glutamatergic motor neurons in the larval neuromuscular system: $1 \mathrm{~b}$ (big boutons) and 1s (small boutons). Larval muscle fibers are divided into four fields: the ventral, ventrolateral, lateral, and dorsal fields. Each $1 \mathrm{~b}$ motor neuron innervates one or two muscle fibers. The three 1 s motor neurons have multiple branches, and each 1 s neuron forms branches on most or all of the fibers within a specific muscle field (Hoang and Chiba, 2001). DIP- $\alpha$ is expressed in MNISN-1s, which synapses on dorsal muscles, and in MNSNb/d-1s, which synapses on ventral and ventrolateral muscles. Fate determination and axon guidance of MNISN-1s have been extensively studied in embryos, where it is known as RP2 (Frasch et al., 1987; Landgraf et al., 1997; Patel et al., 1989; Schmid et al., 1999; Sink and Whitington, 1991).

A subset of muscles innervated by MNISN-1s axon branches are muscles 4, 3, and 2, which are arranged in a ventral $\rightarrow$ dorsal sequence (Figure 1C). In DIP- $\alpha$ mutant larvae, the interstitial axon branch onto muscle $4(\mathrm{~m} 4)$ is always missing, and the branch onto $\mathrm{m} 3$ is usually absent. The branch onto $\mathrm{m} 2$, however, is always present. MNISN-1s filopodia are observed in the $\mathrm{m} 4$ target area in both wild-type and $D I P-\alpha$ mutant embryos, but $1 \mathrm{~s}$ boutons never form on $\mathrm{m} 4$ in mutants. This suggests that nascent axonal projections onto $\mathrm{m} 4$ fail to stabilize and convert into NMJs in the absence of DIP- $\alpha$.

The 'Dpr-ome' revealed that DIP- $\alpha$ binds to Dpr6 and Dpr10. We examined phenotypes in the larval neuromuscular system caused by loss of these Dprs, and found that in $d p r 10$ null mutant larvae the MNISN-1s axon branch onto $\mathrm{m} 4$ is missing, mimicking the DIP- $\alpha$ mutant phenotype. In $3^{\text {rd }}$ instar larvae, dpr10 is expressed in almost all muscle fibers. However, during motor axon outgrowth in embryos, $d p r 10$ expression initiates in two muscle fibers in the immediate vicinity of $\mathrm{m} 4$, and then comes on in $\mathrm{m} 4$ itself around the time at which axon branches appear on this muscle. These results suggest that Dpr10 is a muscle 
recognition cue whose binding to DIP- $\alpha$ on the motor axon triggers recognition and stabilization of the MNISN-1s filopodia on specific muscles.

The accompanying paper shows that DIP- $\alpha$ and Dpr10 have expression patterns in adult leg motor neurons and muscles that are qualitatively similar to those seen in the larval neuromuscular system, and that loss of DIP- $\alpha$ or Dpr10 causes failure of DIP- $\alpha$-expressing leg motor neurons to innervate a subset of their normal muscle targets. Thus, in both of these neuromuscular systems, interactions between DIP- $\alpha$ and Dpr10 control formation of synapses on specific muscle targets.

\section{RESULTS}

\section{$D I P-\alpha$ is selectively expressed by two identified motor neurons}

In a previous study, we showed that several Dprs and DIPs, including DIP- $\alpha$, are expressed in subsets of neurons in the larval ventral nerve cord (VNC). DIP- $\alpha$-GFP reporter expression was observed in a subset of neurons (Figure S1B), including a segmentally repeated pair of motor neurons (Carrillo et al., 2015). Here we sought to investigate the identity of these DIP- $\alpha$-expressing neurons and to determine if DIP- $\alpha$ is required for their targeting to specific muscles. We monitored DIP- $\alpha$ expression with reporters driven by a gene trap GAL4 in embryos and third instar larvae (Figures 1A, B, respectively). The GAL4 line was generated by replacing a splice-trap MiMIC transposable element, MI02031 (Venken et al., 2011) in the first DIP- $\alpha$ coding intron with a T2A-GAL4 cassette (Diao and White, 2012), using recombination-mediated cassette exchange (RMCE). It has been demonstrated that most MiMIC-derived GAL4 cassettes faithfully reproduce the expression patterns of the genes into which they are inserted (Nagarkar-Jaiswal et al., 2015b). T2AGAL4 cassettes in coding introns have the additional feature that GAL4 expression is from a transcript whose translation initiates at the normal ATG of the gene. This means that the expression pattern of GAL4 should correspond to that of the nascent endogenous protein. However, proteins are also subject to post-translational control, so GAL4-driven reporter expression may not necessarily mimic the expression pattern of an accumulated protein as observed by antibody staining (Diao et al., 2015; Venken et al., 2011).

The first cells to express the DIP- $\alpha$-T2A-GAL4>UAS-EGFP reporter (DIP- $\alpha>E G F P)$ include a pair of segmentally repeated neurons in the stage 14 (st14) embryonic VNC (Figure S3A). $D I P-\alpha$ expression persists into late embryonic development, and the segmentally repeated pair of dorsal DIP- $\alpha+$ cells also express the transcription factor Even-skipped (Eve) by st16 (Figure 1A). The three prominent medially located pairs of Eve+ neurons correspond to the well-characterized aCC, pCC, and RP2 neurons (Landgraf et al., 1997; Patel et al., 1989; Schmid et al., 1999). pCC is an interneuron, and aCC is the Ib-type motor neuron that innervates muscle 1 (m1). RP2, known as MNISN-1s during larval development (Figure 1B), innervates the dorsal muscle field. Based on the stereotyped positions of the neuronal cell bodies in the VNC that express $D I P-\alpha$, we conclude that $D I P-\alpha$ is selectively expressed in MNISN-1s/RP2 (hereafter referred to as MNISN-1s). 
In order to prove that the medial $D I P-\alpha+$ cell is MNISN-1s, we examined its muscle innervation pattern in third instar larvae. Motor neurons make stereotyped connections with their muscle targets, allowing us to utilize the innervation pattern of the DIP- $\alpha+$ Eve+ neuron to identify it. Using the same $D I P-\alpha>E G F P$ reporter, we observe two axons that exit the VNC in each hemisegment. One of these axons innervates the dorsal muscles (Figure 1C), corresponding to the known connectivity map of MNISN-1s (Hoang and Chiba, 2001). The other axon innervates ventral and ventrolateral muscles, corresponding to the connectivity map of MNSNb/d-1s (Figure S1C). The MNSNb/d-1s cell body is located ventrally within the VNC (Figure S1A) and is not Eve+. When NMJs on all muscles are visualized with anti-HRP, it is observed that only 1 s boutons express the DIP- $\alpha>E G F P$ reporter. Not all 1s boutons are labeled with the reporter, however. There is a third 1s motor neuron that innervates lateral muscles, and this does not express DIP- $\alpha$. In summary, $D I P-\alpha$ is expressed by two of the three 1 s motor neurons in each abdominal hemisegment, and not by any $1 \mathrm{~b}$ motor neurons.

DIP- $\alpha$ is a cell surface protein, and analysis of its subcellular localization might provide insights into its function. To address where DIP- $\alpha$ localizes within MNISN-1s, we used a 'protein trap', DIP- $\alpha$-GFP-DIP- $\alpha$, constructed by using RMCE to insert GFP into the reading frame of the same MiMIC as for the T2A-GAL4 replacement (Nagarkar-Jaiswal et al., 2015a). If such protein traps are expressed well, they usually are transported to the subcellular compartments where the endogenous protein is located. We observed that DIP- $\alpha-G F P-D I P-$ $\alpha$ localized in a punctate distribution to the NMJ, and specifically within MNISN-1s boutons (Figure 1D). We were unable to determine if DIP- $\alpha$-GFP-DIP- $\alpha$ also localizes to MNISN-1s dendrites due to the abundant signal in the VNC neuropil (Figure S1D).

\section{DIP- $\alpha$ is required for MNISN-1s targeting specificity}

Previously, we and others reported expression patterns that support a role for Dpr-DIP interactions in synaptic partner choice (Carrillo et al., 2015; Tan et al., 2015). Given the selective expression of $D I P-\alpha$ in two 1s motor neurons within the neuromuscular system and the localization of DIP- $\alpha$ to presynaptic terminals, we speculated that DIP- $\alpha$ may be involved in targeting of motor axons to muscle fibers. The innervation pattern of the larval musculature is almost invariant, so we can readily evaluate changes in connectivity due to alterations in gene function. MNISN-1s innervates most of the dorsal muscles, including muscles 1, 2, 3, 4, 9, 10, 19, and 20 (Hoang and Chiba, 2001).

The DIP- $\alpha$-GAL4 reporter that we used for expression studies is also an LOF allele, since the splice-trap into the GAL4 cassette creates a truncated DIP- $\alpha$. Conveniently, due to the fact that $D I P-\alpha$ is an $\mathrm{X}$-linked gene, we could use $D I P-\alpha>E G F P /+$ heterozygous females as controls and DIP- $\alpha>E G F P / Y$ hemizygous males as LOF mutants. In controls, the MNISN-1s branch onto $\mathrm{m} 4$, hereafter referred to as $\mathrm{m} 4-1 \mathrm{~s}$, is easily identifiable and reproducibly found (Figure 2A). Remarkably, loss of DIP- $\alpha$ produces a unique phenotype in which the MNISN-1s branch on $\mathrm{m} 4$ is always missing (the m4-1s phenotype; Figure 2B). In control $D I P-\alpha$ heterozygous animals, MNISN-1s innervates $m 4$ with a frequency of $77 \%$, but we never observed m4-1s branches in DIP- $\alpha>E G F P$ hemizygous males (Figure 2B, D). We confirmed the loss of innervation with a targeted null deletion mutation in $D I P-\alpha\left(D I P-\alpha^{1-}\right.$ 
178; Figure 2D) produced by CRISPR. Thus, DIP- $\alpha$ is necessary for innervation of $m 4$ by MNISN-1s.

\section{DIP- $\alpha$ is required presynaptically for proper MNISN-1s connectivity}

Although DIP- $\alpha$ reporters demonstrate that DIP- $\alpha$ is expressed in MNISN-1s and localizes to MNISN-1s terminals, we cannot rule out low-level expression in muscles. To define the cells in which $D I P-\alpha$ is required for proper MNISN-1s innervation, we performed rescue experiments by expressing a C-terminally Myc-tagged UAS-DIP- $\alpha$ construct in a DIP- $\alpha$ mutant background. First, we confirmed that the tagged DIP- $\alpha$ is able to localize to MNISN1s NMJs on $\mathrm{m} 4$ by staining with anti-Myc and anti-DIP- $\alpha$ antibodies (Figure S2A, B). The lack of DIP- $\alpha$ in the MNISN-1s axon in the intersegmental nerve (Figure S2A, B) also suggests that DIP- $\alpha$ is actively targeted to the NMJ. We then performed GOF controls to ensure that overexpression of DIP- $\alpha$ in MNISN-1s had no adverse effects on targeting. Overexpression of DIP- $\alpha$ utilizing two drivers that are expressed in subsets of motor neurons (eve $e^{R N 2}-G A L 4$, expressed in MNISN-1s and aCC, plus interneurons; and DIP- $\alpha-G A L 4$, expressed in MNISN-1s and MNSNb/d-1s, plus interneurons) produced normal m4-1s NMJs. We expressed these same constructs in a DIP- $\alpha$ null background and found complete rescue of the m4-1s phenotype (Figure 2C,D), suggesting that DIP- $\alpha$ functions in MNISN-1s. We confirmed that DIP- $\alpha$ is required in MNISN-1s using RNAi-mediated knock down of DIP- $\alpha$. Utilizing either Elav-GAL4 (pan-neuronal) or DIP- $\alpha$-GAL4 to drive RNAi expression, we observed significant reductions in the frequency of m4-1s branches (Figure 2F).

Knockdown of DIP- $\alpha$ in muscles using Mef2-GAL4-driven RNAi had no effect on m4-1s, indicating that DIP- $\alpha$ is required only in the presynaptic neuron.

Interestingly, driving pan-neuronal expression of DIP- $\alpha$ with Elav-GAL4 in a wild-type background produced a complete loss of m4-1s, similar to DIP- $\alpha$ LOF. The same result was observed when DIP- $\alpha$ was expressed in muscles using Mef2-GAL4 (Figure 2E). Possible models to explain these surprising results are considered in the Discussion.

\section{DIP- $\alpha$ does not control MNISN-1s axon guidance or defasciculation}

Motor neuron axons exit the VNC during embryonic st13-14 and begin to innervate their muscle targets prior to hatching (Sanchez-Soriano et al., 2007). During the larval instars, NMJs formed during embryogenesis expand in order to keep pace with the growth of the muscle fibers. We investigated whether DIP- $\alpha$ is expressed at the appropriate embryonic stage to function in MNISN-1s muscle targeting. MNISN-1s begins to express DIP- $\alpha>E G F P$ at st13/14 (Figure S3A) and this expression continues through embryonic st17 and then persists during larval development (Figure 1). The proper wiring of MNISN-1s requires the coordination of axon guidance, fasciculation, defasciculation, and synaptic partner choice. Thus, the striking loss of m4-1s observed upon removal of DIP- $\alpha$ could result from defects in various developmental processes.

Axon guidance requires the integration of attractive and repulsive external cues through cell surface proteins. MNISN-1s innervates the dorsal muscles, the most distal of which is $\mathrm{m} 1$. The MNISN-1s axon reaches its terminus near the proximal edge of $\mathrm{m} 1$ before it forms a branch on m4. Thus, if DIP- $\alpha$ is required for axon guidance we would expect to observe defects in MNISN-1s axons in the vicinity of $\mathrm{m} 1$ and $\mathrm{m} 2$ in mutants. In control st15 embryos, 
the MNISN-1s growth cone is easily identifiable in the $\mathrm{m} 1 / \mathrm{m} 2$ target area (Figure $3 \mathrm{~A}$ ). DIP$\alpha$ nulls are indistinguishable from controls, suggesting that axon guidance is likely not affected (Figure 3B). Additionally, in third instars, we observe proper MNISN-1s innervation of $\mathrm{m} 2$ in $D I P-\alpha$ mutants (Figure 4 ).

Another critical step in establishing neuromuscular wiring is the defasciculation of motor neuron axons from the main nerve. This process requires the appropriate balance between adhesion between axons and repulsive signals that facilitate separation of axons from the nerve bundle. Defasciculation is typically initiated by filopodia emanating from the growth cone (when an entire axon leaves the bundle) or from the axon shaft (when an interstitial branch is being formed). We investigated whether DIP- $\alpha$ was required for MNISN-1s filopodia to leave the nerve bundle. In control st17 embryos, there are obvious motor neuron filopodia, including MNISN-1s filopodia (identified by their selective expression of $D I P-\alpha-G A L 4>E G F P$ ) that defasciculate from the ISN throughout the dorsal body wall (Figure 3C). We observed similar filopodial projections in age-matched DIP- $\alpha$ null embryos (Figure $3 \mathrm{D}$ caret), ruling out a function for DIP- $\alpha$ in general defasciculation (Figure 3C,D asterisk).

We then looked more closely at MNISN-1s axon defasciculation and bouton formation at $\mathrm{m} 4$, since this would address whether the m4-1s branch initially forms and then retracts or never forms at all in DIP- $\alpha$ mutants. During axon pathfinding, filopodia extend and contact both target and non-target muscles. The developmental time course for MNISN-1s innervation of $\mathrm{m} 4$ is not known, so we examined MNISN-1s axons at various embryonic stages and in $1^{\text {st }}$ instar larvae. In control embryos, we observe MNISN-1s axon defasciculation and filopodia at m4 beginning in st17 (Figure 3C). MNISN-1s filopodia at more dorsal muscles are evident at earlier stages (Figure 3A). In DIP- $\alpha$ LOF st17 embryos, we still observe MNISN-1s axon protrusions at m4 (Figure 3D), suggesting that the m4-1s branch initially extends but is not properly stabilized due to loss of $D I P-\alpha$. In first instar larvae, $1 \mathrm{~s}$ boutons are found on $\mathrm{m} 4$ in wild-type (Figure 3E), but we never observed a $1 \mathrm{~s}$ bouton on $\mathrm{m} 4$ in DIP- $\alpha$ mutants (Figure 3F; 64 hemisegments examined). These data suggest that MNISN-1s filopodia still contact muscle 4 in the absence of DIP- $\alpha$, but fail to establish a stable interstitial branch.

\section{Loss of DIP- $\alpha$ selectively reduces and expands MNISN-1s connectivity}

The localization of DIP- $\alpha$ to all MNISN-1s boutons prompted us to perform a more exhaustive analysis of MNISN-1s targeting to determine if there were any subtle changes in connectivity. Previous studies reported that MNISN-1s innervates 8 dorsal muscles $(1,2,3$, $4,9,10,19$, and 20) (Hoang and Chiba, 2001). A detailed analysis of innervation shows that MNISN-1s targets m2, 3, 4, 9, 10, 19, and 20 almost invariantly (Figure 4A,C). However, m1, the most dorsal muscle, is usually not innervated in control animals.

We repeated this analysis in a DIP- $\alpha$ null background and observed interesting alterations in connectivity. First, $\mathrm{m} 1$, which rarely has 1 s innervation, consistently had 1s branches (Figure 4B,C). Second, two muscles, m11 and m18, which are never innervated by MNISN1 in wild-type, now sometimes receive ectopic 1 s branches upon removal of DIP- $\alpha$. Third, muscles adjacent to $\mathrm{m} 4$ also lose 1 s innervation in mutants (Figure 4B,C). Specifically, $\mathrm{m} 3$, 19 , and 20 show major reductions in 1 s branch frequency. It is interesting to note that the 
more dorsal muscles in the dorsal muscle field gain 1 s branches $(\mathrm{m} 1,11,18)$, while more ventral muscles $(\mathrm{m} 3,4,19,20)$ lose innervation.

Expression of DIP- $\alpha$ binding partners, Dpr6 and Dpr10, in the neuromuscular system DIP- $\alpha$ binds to only two of the 21 Dprs, Dpr6 and Dpr10 (Ozkan et al., 2013). We sought to determine where $d p r 6$ and $d p r 10$ are expressed, in order to ascertain if either could potentially function with DIP- $\alpha$ to control MNISN-1s targeting. dpr6-T2A-GAL4>EGFP shows dpr6 expression in subsets of cells in the embryonic VNC, including MNISN-1s and aCC (Figure 5A), and analysis of the larval bodywall reveals that dpr6 is expressed in type $1 \mathrm{~b}$ and 1s motor neurons (Figure 5B). Thus, $d p r 6$ and DIP- $\alpha$ are co-expressed in MNISN-1s.

We next investigated $d p r 10$ expression using a $d p r 10-T 2 A-G A L 4$ reporter. $d p r 10$ is found in subsets of cells in the embryonic VNC (Fig. S4) but, unlike $d p r 6$ and DIP- $\alpha$, it is not expressed in embryonic MNISN-1s (Figure 5C). $d p r 10$ is prominently expressed in third instar larval muscles, including $\mathrm{m} 4$ (Figure 5D). As the GFP signal is very high within muscles, we were unable to determine if $d p r 10$ was expressed in motor neurons with the current reporter. Some signal is detected in the nerves (Figure 5D caret), suggesting that $d p r 10$ is expressed in neurons that have axons in the periphery. To define Dpr10 localization, we expressed a UAS-Dpr10-V5 construct in muscles. Remarkably, V5 staining was selectively found at NMJs, surrounding both $1 \mathrm{~b}$ and $1 \mathrm{~s}$ boutons, indicating that the protein localizes to the postsynaptic side of NMJs (Figure 5E).

\section{Dpr10 is able to bind DIP- $\alpha$ in tissue}

Based on DIP- $\alpha$ localization to MNISN-1s terminals, we hypothesize that DIP- $\alpha$ interacts in trans with another cell surface protein on $\mathrm{m} 4$ to confer specificity. An attractive candidate for this role is Dpr10, which is expressed in muscles and interacts biochemically with DIP- $\alpha$. We sought to determine if DIP- $\alpha-$ Dpr10 interactions could occur in the larval tissue. We used a recombinant DIP- $\alpha$ fused with pentameric alkaline phosphatase (DIP- $\alpha$-AP5) to probe dissected third instar larvae. The DIP- $\alpha$-AP5 was then detected by

immunohistochemistry with antibodies against the AP epitope. In wild type animals, DIP- $\alpha$ AP5 shows minimal binding to tissue (Figure 5F). However, upon ectopic postsynaptic expression of Dpr10-V5 in muscles, we observed strong labeling of the postsynaptic membrane surrounding boutons (Figure 5G), suggesting that DIP- $\alpha$ is able to directly bind to Dpr10. This observation supports a model whereby endogenous muscle Dpr10 interacts transsynaptically with DIP- $\alpha$ in MNISN-1s terminals.

\section{dpr10 is required for MNISN-1s innervation of M4}

Since Dpr10 interacts with DIP- $\alpha$, both in vitro and in tissue, and is able to localize to the postsynaptic membrane, we decided to investigate the requirement of $d p r 10$ for MNISN-1s innervation of $\mathrm{m} 4$. In order to distinguish $1 \mathrm{~s}$ and $1 \mathrm{~b}$ terminals, we stained for a subsynaptic reticulum (SSR) marker, Discs-Large (Dlg), and identified 1s boutons by their less extensive SSR relative to $1 \mathrm{~b}$ boutons (Guan et al., 1996) (Figure 6A). We examined a CRISPRgenerated null deletion mutant of $d p r 10, d p r 10^{14-5}$. In this mutant, we observed loss of almost $100 \%$ of 1 s boutons on $\mathrm{m} 4$ (Figure 6B and D), similar to the DIP- $\alpha$ phenotype (Figure 2B). 1b boutons were still present on $\mathrm{m} 4$ (Figure 6B), indicating that Dpr10 is specifically required for MNISN-1s innervation. 
Since $d p r 6$ is expressed in $1 \mathrm{~b}$ and 1 s motor neurons, and $D I P-\alpha$ is in 1 s motor neurons, whose axons fasciculate with $1 \mathrm{~b}$ axons, axon-axon contact through Dpr6-DIP- $\alpha$ interactions could potentially affect MNISN-1s targeting to m4. However, as shown above, MNISN-1s is able to properly defasciculate without DIP- $\alpha$ (Figure 3C, D). Also, dpr6-T2A$G A L 4 / D f$ mutants have no m4-1s phenotype, and do not produce any other detectable alterations in MNISN-1s (Figure 6C and D). Thus, the role of DIP- $\alpha$ in formation of the m41s branch is likely to be mediated through its interactions with Dpr10.

In the neuromuscular system, we found that $d p r 10$ is expressed in neurons (Carrillo et al., 2015) and in muscles (Figure 5D). To determine which cells require Dpr10 function for normal formation of the m4-1s branch, we used cell type specific RNAi knockdown of $d p r 10$. Presynaptic expression of $d p r 10$-RNAi with DIP- $\alpha$-Gal4 did not affect MNISN-1s targeting to m4 (Figure 6F). However, driving dpr10-RNAi in muscles (Mef2-Gal4) phenocopied the dpr10 null mutation, producing an almost complete loss of m4-1s branches (Figure 6F). This result indicates that $d p r 10$ is required in muscles. Interestingly, pan-neuronal expression of $d p r 10$-RNAi produced a partial loss of m4-1s (Figure 6F), suggesting that Dpr10 expression in a non-1s neuron also contributes to proper targeting.

We also attempted to rescue the m4-1s phenotype using the UAS-Dpr10-V5 construct described above. Dpr10-V5 localizes to NMJs when it is expressed in muscles. However, we observed that expression of Dpr10-V5 from pan-neuronal (Elav-GAL4) or muscle (Mef2GAL4) drivers reduced the frequency of m4-1s branches, even in a wild-type background (Figure 6E), similar to DIP- $\alpha$ GOF. Pan-neuronal expression of Dpr10 almost eliminated m4$1 \mathrm{~s}$ branches. We asked whether this was likely to be a cis or trans effect by expressing Dpr10 only in 1s neurons. We observed that expression of Dpr10-V5 in MNISN-1s using $D I P-\alpha$-GAL4 also produced loss of M4-1s branches (Figure 6E), suggesting that a GOF cis DIP- $\alpha-D$ pr10 interaction can interfere with the DIP- $\alpha-D$ pr10 trans interactions that are important for branch formation.

\section{dpr10 is dynamically expressed in muscle subsets during embryonic development} In third instar larvae, $d p r 10$ is expressed in almost all abdominal muscles, but loss of branches in DIP- $\alpha$ or $d p r 10$ mutants is only observed for $\mathrm{m} 4$ and adjacent muscles. This led us to examine if $d p r 10$ expression in muscles was temporally and spatially regulated during the period of embryonic development in which the neuromuscular circuit is established. We used the $d p r 10>E G F P$ reporter and focused on three stages: late 14/early 15, late 15/early 16, and late 16/early 17. Using confocal images of embryos stained for the axonal marker Fasciclin II (Fas2; used to more accurately determine developmental stage) and GFP, we qualitatively scored samples, where muscle GFP signal above background received a score of 1 , and anything at background levels received a score of 0 . Figure $7 \mathrm{G}$ thus represents the probability that $d p r 10>E G F P$ is expressed in each muscle examined.

The first muscles that reproducibly show dpr10 expression are m5 and m20, which flank m4 (Figure 7A, B and G). In late st15/early st16 embryos, dpr10 expression becomes more consistent in $\mathrm{m} 5$ and $\mathrm{m} 20$ and expands to $\mathrm{m} 2$ in the dorsal muscle field and several 
muscles in the ventral muscle field (Figure 7C, D and G). By late st16/early st17, most muscles, including m4, express $d p r 10$ (Figure 7E-G). The first MNISN-1s filopodia in the vicinity of $\mathrm{m} 4$ are observed in late st16/early st17 embryos, and the first discernable $1 \mathrm{~s}$ boutons on $\mathrm{m} 4$ are found in early first instar larvae (Figure 3). Thus, $d p r 10$ expression in $\mathrm{m} 4$ coincides with MNISN-1s innervation. The earlier expression of $d p r 10$ in muscles adjacent to $\mathrm{m} 4$ ( $\mathrm{m} 5$ and $\mathrm{m} 20$ ) might mean that these muscles provide cues for formation or stabilization of an MNISN-1s axon branch in this area. Innervation of m20 is also lost in $D I P-\alpha$ mutants. $\mathrm{m} 5$, however, lacks 1 in innervation even in wild-type. Interestingly, $\mathrm{m} 18$ and m11 do not express $d p r 10$ even in st17 embryos (Figure 7G). These muscles are among those that are not innervated by either of the DIP- $\alpha$ positive motor neurons (Figure 4C). Some of these muscles do receive $1 \mathrm{~s}$ innervation from the third 1 s motor neuron, which does not express $D I P-\alpha$.

\section{DISCUSSION}

In this paper, we show that interactions between DIP- $\alpha$ and its in vitro binding partner, Dpr10, are essential for innervation of a specific subset of larval muscle fibers by branches of the MNISN-1s motor axon. DIP- $\alpha$ is expressed by only two motor neurons, and the protein localizes to the NMJs of those neurons (Figures 1, S1). MNISN-1s innervates most of the muscles in the dorsal muscle field, but only the proximal (most ventral) branches of its axon are affected in DIP- $\alpha$ mutants. The branch innervating $\mathrm{m} 4, \mathrm{~m} 4-1 \mathrm{~s}$, is absent in $100 \%$ of hemisegments in mutants. DIP- $\alpha$ is required in the MNISN-1s neuron to direct innervation of $\mathrm{m} 4$ (Figure 2). Examination of the MNISN-1s axon during embryonic development shows that its filopodia explore the surface of $\mathrm{m} 4$ and surrounding muscles in a normal manner in $D I P$ - $\alpha$ mutants, but a stable m4 NMJ never forms (Figure 3). Innervation of muscles near $\mathrm{m} 4$ is also reduced in DIP- $\alpha$ mutants, while innervation of more dorsal muscles is increased (Figure 4). One of DIP- $\alpha$ 's two binding partners, Dpr10, is expressed at high levels in muscles and can localize to the postsynaptic side of NMJs (Figure 5), and the m4-1s branch is also absent in $d p r 10$ mutants. RNAi knockdown experiments show that Dpr10 is required in muscles (Figure 6). By examination of the temporal and spatial expression patterns of $d p r 10$ in embryos, we found that its earliest expression is on muscles flanking $\mathrm{m} 4$, some of which also lack 1s NMJs in DIP- $\alpha$ mutants (Figure 7). This suggests that recognition of Dpr10 on these muscles by DIP- $\alpha$ on the MNISN-1s growth cone is a cue for branch formation or stabilization.

\section{A model for control of muscle innervation by interactions between DIP- $\alpha$ and Dpr10}

A number of mutant screens for alterations in the morphologies and patterning of NMJs in the larval neuromuscular system have been performed (Aberle et al., 2002; Kraut et al., 2001; Valakh et al., 2012). LOF mutations in a few genes, including those encoding the cellsurface IgSF domain protein Sidestep and its binding partner, Beaten Path, cause motor axons to fail to arborize normally onto any muscle fibers, resulting in large-scale alterations in innervation patterns (Kinold et al., 2018; Pipes et al., 2001; Siebert et al., 2009; Sink et al., 2001). However, there are no prior reports (to our knowledge) of LOF mutations in single genes that cause high-penetrance changes in targeting of single larval motor axons to individual, or groups of, muscle fibers. 
The failure to find genes required for innervation of specific muscles in LOF screens has suggested that individual muscles may be labeled by multiple targeting cues, and that neurons express receptors for more than one of these cues. Loss of any one neuronal receptor or muscle targeting cue does not cause strong phenotypes because they have partially redundant functions. The remaining receptors and cues may substitute for the missing proteins in mutants and allow normal muscle targeting to occur.

It has been difficult to identify neuronal receptors whose expression is specific to particular subsets of motor axons. Neuronal CSPs that have been previously studied in the context of motor axon guidance and arborization onto muscles (e.g., Receptor Tyrosine Phosphatases (RPTPs), Beaten Paths, Fasciclin II, Netrin receptors, Semaphorin receptors) are usually expressed by most or all motor neurons. Not surprisingly, then, mutations in genes encoding these proteins usually produce guidance or arborization phenotypes that affect many motor axons and muscles. By contrast, DIP- $\alpha$ is expressed in only two of the 35 motor neurons that innervate muscles in each larval abdominal hemisegment. These are the $1 \mathrm{~s}$ motor neurons MNISN-1s (RP2) and MNSNb/d-1s. This finding suggested that any phenotypes caused by loss of DIP- $\alpha$ might be specific to the axons of these two motor neurons.

Like other motor axons, the two DIP- $\alpha$-expressing axons probably express binding partners for many muscle cell-surface proteins. Neuronal and muscle binding partners could act as signaling receptors, ligands for receptors, or adhesion molecules. The 1 s motor axons that express DIP- $\alpha$ have multiple branches, and each axon innervates most of the muscles within a muscle field. MNISN-1s innervates muscles in the dorsal field (Hoang and Chiba, 2001; Landgraf et al., 1997; Nose, 2012). One might expect that targeting phenotypes would be observed in DIP- $\alpha$ LOF mutants only if binding of DIP- $\alpha$ to one of its Dpr binding partners was essential for recognition of specific muscle fibers by individual branches of a 1s motor axon. In fact, we observed that the loss of DIP- $\alpha$ causes a high-penetrance loss of branches onto a particular group of dorsal muscle fibers innervated by MNISN-1s. These are internal muscles 4 and 3, and external muscles 19 and 20, which are underneath m4 and $\mathrm{m} 3$ in embryonic and larval 'fillet' preparations. However, the branches of the MNISN1s axon onto internal dorsal muscle 2 and external muscles 9 and 10, which lie underneath $\mathrm{m} 2$, are still present in $D I P-\alpha$ mutants.

The same MNISN-1s branches are lost with high penetrance in $d p r 10$ null mutants, indicating that Dpr10 is the DIP- $\alpha$ binding partner relevant to innervation of these muscles. In larvae, $d p r 10$ is expressed at high levels in most muscle fibers. Knockdown of $d p r 10$ by RNAi in all muscles affects formation of the same MNISN-1s branches that are eliminated in dpr10 mutants. Therefore, binding of neuronal DIP- $\alpha$ to muscle Dpr10 is likely to underlie recognition of specific muscles as targets for proximal MNISN-1s axon branches. In embryos, $d p r 10$ expression is initiated in m20 and m5, which flank m4. NMJs on m20 are also absent in DIP- $\alpha$ mutants; $\mathrm{m} 5$ does not receive $1 \mathrm{~s}$ innervation. $d p r 10$ expression begins in $\mathrm{m} 4$ around the time at which we observe exploration of this muscle by filopodia emerging from the MNISN-1s axon (Figure 7). 
The gene encoding DIP- $\alpha$ 's other binding partner, Dpr6, is expressed by most motor neurons, but is not detectably expressed by muscle fibers. Although dpr6 is expressed by MNISN-1s in embryos, dpr6 mutants do not have m4-1s phenotypes. This suggests that Dpr6 does not play a direct role in the targeting of MNISN-1s to m4. DIP- $\alpha$ can also bind homophilically, but with reduced affinity relative to its heterophilic binding affinities for Dpr10 and Dpr6 (Cheng et al., 2018). However, during normal development DIP- $\alpha$ would not have the opportunity to mediate homophilic interactions between motor axons, since it is expressed only on the MNISN-1s axon and not on the other motor axons with which it fasciculates during outgrowth.

Correct innervation of $\mathrm{m} 4$ and the other muscles in its immediate vicinity may require a balance between the expression levels of DIP- $\alpha$ 's binding partner Dpr10 on muscles vs. axons. As described above, knocking down Dpr10 in muscles eliminates innervation of $\mathrm{m} 4$ (Figure 6), suggesting that transsynaptic interactions between neuronal DIP- $\alpha$ and muscle Dpr10 are essential for recognition of this muscle by an interstitial MNISN-1s growth cone. There is also a 50\% reduction in m4 innervation when Dpr10 is knocked down in all neurons, while knockdown in MNISN-1s produces no innervation defects. This suggests that interactions between DIP- $\alpha$ on MNISN-1s axons and Dpr10 on other axons with which it fasciculates also contribute to correct $\mathrm{m} 4$ innervation.

Driving high-level expression of DIP- $\alpha$ or Dpr10 in all neurons abolishes $\mathrm{m} 4$ innervation by MNISN-1s. Interestingly, these GOF phenotypes are also seen when Dpr10, but not DIP- $\alpha$, is increased in MNISN-1s only. High-level expression of DIP- $\alpha$ or Dpr10 on muscles also eliminates (DIP- $\alpha$ ) or reduces (Dpr10) innervation of m4. Some of these phenotypes may be due to cis Dpr10-DIP- $\alpha$ interactions on the same membrane, which could reduce the amount of DIP- $\alpha$ or Dpr10 that is available to interact with its partner in trans. Excessive adhesion between the MNISN-1s axon and the other axons in its bundle (in the case of Dpr10 overexpression in all neurons), or between the MNISN-1s axon and the muscles it traverses during its outgrowth (in the case of Dpr10 overexpression in muscles) may also affect the ability of a branch to separate from the axon and form an NMJ.

Knockdown or overexpression of DIP- $\alpha$ or Dpr10 in neurons or in muscles does not reduce the frequency of innervation of the most dorsal muscles by MNISN-1s, indicating that these muscles are recognized as targets via other cues. Interestingly, however, $\mathrm{m} 1$, which is adjacent to $\mathrm{m} 2$ and rarely innervated by MNISN-1s, gains innervation in DIP- $\alpha$ mutants, and the 1s NMJ on $\mathrm{m} 2$ becomes larger. These results suggest that MNISN-1s is normally specified to make a certain number of synaptic boutons, and that loss of boutons on proximal muscles $\mathrm{m} 4, \mathrm{~m} 3, \mathrm{~m} 19$, and $\mathrm{m} 20$ results in an increased number of boutons on distal muscles.

Using these results, we have constructed a model that can explain how interactions between DIP- $\alpha$ and Dpr10 specify targeting of MNISN-1s axon branches to $\mathrm{m} 4$ and the other muscles in its vicinity. DIP- $\alpha$ begins to be expressed in MNISN-1s (RP2) in st14 embryos, during the period of motor axon guidance. The MNISN-1s axon reaches its terminus in the vicinity of $\mathrm{m} 1 / \mathrm{m} 2$ at stage 16 , before it forms interstitial branches onto 
m20, where Dpr10 is already expressed. After the m20-1s branch forms, Dpr10 appears on $\mathrm{m} 4$, and binding of DIP- $\alpha$ on MNISN-1s to Dpr10 on $\mathrm{m} 4$ and surrounding muscles results in the formation of stable branches that differentiate into NMJs. During this process, DIP- $\alpha$ on MNISN-1s might switch from interacting with Dpr10 on fasciculated axons within the ISN bundle to binding to Dpr10 on muscles.

\section{Functions of the Dpr-DIP network in formation of synaptic circuits}

The Dpr-ome binding network was defined by a global in vitro 'interactome' screen for binding interactions among all Drosophila cell-surface and secreted proteins containing three common extracellular domain types: IgSF, Fibronectin Type III, and LRR. There are 21 Dpr proteins, each containing two IgSF domains, 9 DIP proteins, each containing three IgSF domains, and an LRR protein called cDIP that binds to many Dprs and DIPs (Carrillo et al., 2015; Ozkan et al., 2013) .

Analysis of expression of individual $d p r$ and DIP genes revealed remarkable and unprecedented patterns in the larval ventral nerve cord and pupal brain. Each $d p r$ and DIP is expressed by a small and unique subset of interneurons. In the pupal optic lobe, neurons expressing a particular Dpr are often presynaptic to neurons expressing a DIP to which that Dpr binds in vitro (Carrillo et al., 2015; Tan et al., 2015). These findings suggested that DprDIP interactions might be important for formation of synaptic circuits during brain and ventral nerve cord development.

In our earlier work, we examined the expression and function of DIP- $\gamma$ and its binding partner Dpr11. Dpr11 is selectively expressed in 'yellow' R7 photoreceptors, which make the Rh4 rhodopsin, and DIP- $\gamma$ is expressed in a subset of Dm8 amacrine neurons in the optic lobe medulla. Dm8s receive more synapses from R7 than any other neuron. DIP- $\gamma$ is required for survival of the Dm8 neurons that express it (Carrillo et al., 2015). The fact that loss of DIP- $\gamma$ causes loss of brain neurons that express these proteins suggests that DIPDpr interactions can transmit trophic signals. This does not appear to be the case for either DIP in the larval or adult neuromuscular system, however, since there are no missing motor neurons in $D I P-\gamma$ or $D I P-\alpha$ mutants.

The expression patterns of DIP- $\gamma$ and DIP- $\alpha$ suggest that they may be involved in similar processes during optic lobe development. In addition to yellow R7s, Dpr11 is expressed in a subset of motion-sensitive $\mathrm{T} 4$ and $\mathrm{T} 5$ neurons, which synapse onto large cells called Lobula Plate Tangential Cells (LPTCs). Dpr11-expressing T4 and T5 cells project to the layers 1 and 2 of the lobula plate, and DIP- $\gamma$ is expressed in a small number of LPTCs that arborize in those layers (Carrillo et al., 2015). In the optic lobe lamina, L3 and L5 neurons express Dprs 6 and 10, while L2 expresses only Dpr6. These L cells are synaptically connected to Dm4, Dm12, and Dm1 cells in the medulla, which express DIP- $\alpha$ (Tan et al., 2015).

In the larval neuromuscular system, however, the functions of DIP- $\gamma$ appear to be very different from those of DIP- $\alpha$. DIP- $\gamma$ and Dpr11 are both expressed by most or all motor neurons. In $D I P-\gamma$ and $d p r 11$ LOF mutants, there are no alterations in muscle targeting, but NMJs have phenotypes characterized by the presence of small clustered boutons called 
satellites. Retrograde BMP signaling is upregulated in these mutants (Carrillo et al., 2015). By contrast, DIP- $\alpha$ is expressed by only two motor neurons, and its interactions with Dpr10 expressed on muscles control formation and/or targeting of a specific set of interstitial axon branches.

The functions of DIP- $\alpha$ and Dpr10 appear to be conserved between the larval neuromuscular system and the adult leg neuromuscular system. The accompanying paper from Richard Mann's group shows that DIP- $\alpha$ is expressed in a subset of motor neurons that innervate specific leg muscles, while Dpr10 is expressed in muscles. In DIP- $\alpha$ and dpr10 mutants, the axonal branches onto the muscles targeted by the DIP- $\alpha$-expressing axons are absent. In summary, Dpr10 appears to be one of the long-sought targeting cues that direct recognition of specific muscle fibers as targets, while DIP- $\alpha$ is the corresponding receptor on the motor neurons that innervate these muscles.

\section{METHODS}

Fly strains:

\begin{tabular}{|l|l|}
\hline Fly strain & Source \\
\hline$w^{1118}$ & Bloomington Drosophila Stock center (BDSC) \\
\hline Mef2-GAL4 & Gift of Hugo Bellen \\
\hline$D I P-\alpha-T 2 A-G A L 4$ & Gift of Hugo Bellen \\
\hline$D I P-\alpha-E G F P-D I P-\alpha$ & Gift of Hugo Bellen \\
\hline$D I P-\alpha^{1-178}$ & Gift of Lawrence Zipursky \\
\hline$d p r 10^{14-5}$ & Gift of Lawrence Zipursky \\
\hline UAS-DIP- $\alpha-$ Myc & Gift of Lawrence Zipursky \\
\hline UAS-Dpr10-V5 & Gift of Lawrence Zipursky \\
\hline$d p r 10-T 2 A-G A L 4$ & Gift of Hugo Bellen \\
\hline$d p r 6-T 2 A-G A L 4$ & Gift of Hugo Bellen \\
\hline UAS-2XEGFP & BDSC \#6874 \\
\hline Elav-GAL4 & BDSC \#8765 \\
\hline $24 B-G A L 4$ & BDSC \#1767 \\
\hline$M H C-G e n e S w i t c h$ & Gift of Haig Keshishian \\
\hline Eve $e^{R 2}-G A L 4$ & BDSC \#7470 \\
\hline UAS- $d p r 10-$ RNAi & Vienna Drosophila Resource Center \#103511 \\
\hline UAS- DIP- $\alpha$-RNAi & BDSC \#38965 \\
\hline
\end{tabular}

\section{Transgenic and T2A-Gal4 lines}

For construction of UAS-DIP- $\alpha$-Myc , the Myc sequence (gaacaaaaacttatttctgaagaagatctg) was inserted two amino acids before the end of DIP- $\alpha$ protein sequence, following a GGS linker sequence, and cloned into JFRC28 vector. The final construct was injected into BDSC stock \#9744, and inserted into chromosome 3R via PhiC31 mediated integration (Bestgene, Inc.). Plasmid and primer design were carried out using the software Snapgene. 
MiMIC>T2A-GAL4 lines were generated as described in (Diao et al., 2015). Briefly, flies carrying the MiMIC insertion were crossed with the flies bearing the triplet donor (T2AGAL4 for all three phases of DNA). The $\mathrm{F}_{1}$ males from this progeny carrying both components were then crossed to females carrying germline transgenic sources of Cre and $\Phi C 31$. The $\mathrm{F}_{2}$ males with all four components were crossed to a UAS-2XEGFP reporter line and progeny larvae were screened for T2A-GAL4 transformants. The GFP+ larvae were confirmed by PCR.

Antibodies and fluorescent proteins:

\begin{tabular}{|l|l|l|}
\hline Antibody & Concentration & Source \\
\hline Goat anti-HRP-TRITC & $1: 50$ & $\begin{array}{l}\text { Jackson Immunological Research } \\
\text { (Jackson) \#123-025-021 }\end{array}$ \\
\hline Goat anti-HRP-Alexa405 & $1: 50$ & Jackson \#123-475-021 \\
\hline Mouse anti-Dlg & $1: 100$ & $\begin{array}{l}\text { Developmental Studies Hybridoma Bank } \\
\text { (DSHB) \#4F3 }\end{array}$ \\
\hline Mouse anti-Fas2 & $1: 100$ & DSHB \#1D4 \\
\hline Mouse anti-DIP- $\alpha$ & $1: 20$ & Gift of Lawrence Zipursky \\
\hline Mouse anti-V5 & $1: 400$ & ThermoFisher \#R960-25 \\
\hline Rabbit anti-GFP & $1: 1000$ & ThermoFisher \#A11122 \\
\hline Rabbit anti-Dlg & $1: 40,000$ & Budnik Lab \\
\hline Rabbit anti-Myc & $1: 200$ & Cell Signaling Technology \#71D10 \\
\hline Goat anti-Mouse-Alexa488 & $1: 500$ & ThermoFisher \#A11029 \\
\hline Goat anti-Mouse-Alexa568 & $1: 500$ & ThermoFisher \#A11031 \\
\hline Goat anti-Rabbit-Alexa488 & $1: 500$ & ThermoFisher \#A11008 \\
\hline Goat anti-Rabbit-Alexa568 & $1: 500$ & ThermoFisher \#A11036 \\
\hline Phalloidin-Alexa647 & $1: 100$ & ThermoFisher \#A22287 \\
\hline $\begin{array}{l}\text { Rabbit anti-alkaline } \\
\text { phosphatase }\end{array}$ & $1: 100$ & Abcam \#ab16695 \\
\hline
\end{tabular}

\section{Dissection and Immunocytochemistry:}

Embryonic dissections were performed as in (Lee et al., 2009). Egg laying chambers were setup with adult flies and grape juice plates (3\% agar, $1.3 \%$ sucrose, $25 \%$ grape juice in water) and left in the dark at room temperature to lay eggs for 2 hours. Embryos were incubated overnight at $18^{\circ} \mathrm{C}$, and then raised to $29^{\circ} \mathrm{C}$ for two hours to maximize GFP expression. Under a Zeiss V20 stereoscope with fluorescence embryos were transferred to a microscope slide with double sided tape and staged using the autofluorescence and shape of the gut (Bownes, 1975; Hartenstein et al., 1987). Embryos were dechorionated on the double sided tape with a sharpened metal probe and transferred to a small agar slab. A dissection chamber was then prepared on Superfrost Plus slides (ThermoFisher \#22-037246) by outlining a rectangle with a PAP pen (Research Products International, \#195506) to create a dissecting area. Embryos were transferred to a small piece of double sided tape placed into the dissection area, and then covered with phosphate buffered saline (PBS) (0.01M Sodium Phosphate, $150 \mathrm{mM}$ Sodium Chloride). Using a $0.1 \mathrm{~mm}$ electrolytically sharpened tungsten wire held in a pin vice, the embryos were carefully opened along their dorsal surface, and removed from the vitellin membrane. Dissected embryos were then 
trasferred to the adherent charged slide. When all embryos were dissected, the well was washed once with PBS, and then fixed for 1 hour at room temperature using 4\% paraformaldehyde (20\% paraformaldehyde solution (Electron Microscopy Sciences) diluted into PBS). After fixation, samples were washed with three times, 15 minutes each, in $0.05 \%$ PBST (PBS with $0.05 \%$ TritonX100), and then blocked for 1 hour in $1 \%$ normal goat serum ( $1 \%$ Goat serum in $0.05 \%$ PBST). Slides were incubated in primary antibody solutions overnight at $4^{\circ} \mathrm{C}$ in a humidified chamber. Samples were then washed three times, 15 minutes each, with PBST, and incubated for two hours at room temperature with secondary antibodies. After incubation, samples were washed three times, 15 minutes each, with PBST and mounted in vectashield (Vector Laboratories).

Larval dissections were performed as in (Menon et al., 2015). Wandering third instar larvae were dissected in PBS on Sylgard dishes, and pinned down using $0.1 \mathrm{~mm}$ Insect Pins (FST \#26002-10). Samples were then fixed for 30 minutes using 4\% paraformaldehyde (see above). After fixation samples were washed three times, 15 minutes each, in PBST. Samples were then blocked for 1 hour in $1 \%$ normal goat serum ( $1 \%$ Goat serum in $0.05 \%$ PBST), and then incubated in primary antibodies overnight at $4^{\circ} \mathrm{C}$. On the second day, the larval preps were treated as embryo samples above. All larval washes and antibody incubations were performed with mild agitation on a nutator.

For in vivo staining of larvae (Figure $5 \mathrm{G}, \mathrm{H}$ ), we modified a previously described embryo protocol in (Bali et al., 2016; Fox and Zinn, 2005; Lee et al., 2013). Supernatant from S2 cell culture containing the DIP- $\alpha-\mathrm{AP}_{5}$ fusion protein was concentrated 5 -fold (Amicon Ultra-4 Filter Unit, $100 \mathrm{kDa}$ cutoff), and this concentrate was used directly to stain third instar larvae. After larvae were dissected in sylgard dishes, the area surrounding each larva was patted dry with a Kimwipe. The DIP- $\alpha-\mathrm{AP}_{5}(75 \mu \mathrm{l})$ was applied to each prep and incubated for 1.5 hours at room temperature in a humidified chamber. Following incubation, the standard larval immunocytochemistry protocol (above) was followed.

\section{Microscopy and analysis}

Third instar samples that were quantified for $1 \mathrm{~s}$ bouton presence or absence were examined using a Zeiss AxioImager M2 and a 40X plan-neofluar 1.3NA objective. Boutons were examined using HRP and scored as $1 \mathrm{~s}$ or $1 \mathrm{~b}$ based on Dlg signal, as $1 \mathrm{~s}$ boutons have a smaller and dimmer Dlg signal than $1 \mathrm{~b}$ boutons. For DIP- $\alpha$-Gal4>GFP studies, 1 s boutons were scored based on the presence of GFP although Dlg staining was also present. All imaging was done on a Zeiss LSM800 confocal microscope with 20X plan-apo 0.8NA objective, 40X plan-neofluar 1.3NA objective, or 63X plan-apo 1.4NA objective. All samples were imaged under similar conditions. Some embryo images were imaged using a Zeiss LSM880 with Airyscan to improve resolution of small axonal processes.

For $d p r 10$-Gal4>EGFP embryonic analysis, LSM800 confocal images were acquired of Fas2 (to visualize embryonic stage and nerve processes), GFP (to confirm Dpr10 expression), and Phalloidin (to visualize the muscles). Embryonic stage was determined based on the number of Fas 2 positive longitudinal nerves on either side of the ventral nerve cord midline (1-1.5 longitudinal nerves $=$ st14/15, 1.5-2 = st15/16, 2.5-3 = st16/17).Each muscle was scored 1 or 0 based on the presence or absence of GFP expression, respectively, and 
the resulting numbers pooled into a table. The mean of each muscle subtype was then calculated, and the resulting numbers plotted in a heat map using Prism 7 software (Graphpad Software).

All statistical analysis was performed using Prism 7 software (Graphpad), for multiple comparisons one-way ANOVA analysis was performed.

\section{Acknowledgements}

We thank Larry Zipursky for sharing unpublished $d p r 10$ and DIP- $\alpha$ CRISPR and UAS transgenic lines and Hugo Bellen for dpr6-T2A-GAL4, dpr10-T2A-GAL4, and DIP- $\alpha$-T2A-GAL4. Transgenic DIP- $\alpha$-RNAi stock was obtained from the Vienna Drosophila Resource Center (VDRC, www.vdrc.at). Stocks obtained from the Bloomington Drosophila Stock Center (NIH P400D018537) were used in this study. The (monoclonal antibodies: 4F3, 1D4) developed by [Goodman, C., University of California, Berkeley] were obtained from the Developmental Studies Hybridoma Bank, created by the NICHD of the NIH and maintained at The University of Iowa, Department of Biology, Iowa City, IA 52242.We thank Larry Zipursky and Meike Lobb-Rabe for helpful discussions and comments. This work was supported by National Institutes of Health Grants K01 NS102342 (to R.A.C), R01 NS096509 (to K.G.Z), and R01 NS097161 (subcontract to K.Z. from Engin Özkan).

\section{Author Contributions}

J.A., V.S., and R.A.C. analyzed expression and phenotypes in larvae. J.A. and R.A.C. performed the embryo experiments. L.T, S.X., and Q.X. constructed $d p r 10$ and DIP- $\alpha$ CRISPR lines and UAS-Dpr10 and UAS-DIP- $\alpha$ lines. S.N.-J. generated T2A-GAL4 lines. R.A.C., K.Z, J.A., and V.S. analyzed the data. R.A.C. and K.Z. supervised experiments.

\section{Competing Interests Statement}

The authors declare no competing interests.

\section{References}

Aberle, H., Haghighi, A.P., Fetter, R.D., McCabe, B.D., Magalhaes, T.R., and Goodman, C.S. (2002). wishful thinking encodes a BMP type II receptor that regulates synaptic growth in Drosophila. Neuron 33, 545-558.

Bali, N., Lee, H.K., and Zinn, K. (2016). Live Staining of Drosophila Embryos with RPTP Fusion Proteins to Detect and Characterize Expression of Cell-Surface RPTP Ligands. Methods Mol Biol 1447, 373-384.

Bownes, M. (1975). A photographic study of development in the living embryo of Drosophila melanogaster. J Embryol Exp Morphol 33, 789-801.

Broadie, K.S., and Bate, M. (1993). Development of the embryonic neuromuscular synapse of Drosophila melanogaster. J Neurosci 13, 144-166.

Carrillo, R.A., Ozkan, E., Menon, K.P., Nagarkar-Jaiswal, S., Lee, P.T., Jeon, M., Birnbaum, M.E., Bellen, H.J., Garcia, K.C., and Zinn, K. (2015). Control of Synaptic Connectivity by a Network of Drosophila IgSF Cell Surface Proteins. Cell 163, 1770-1782. 
Cheng, S., Ashley, J., Kurleto, J.D., Park, Y.J., Carrillo, R.A., and Özkan, E. (2018). Molecular Basis of Synaptic Specificity by Immunoglobulin Superfamily Receptors in Drosophila. submitted.

Chiba, A., Snow, P., Keshishian, H., and Hotta, Y. (1995). Fasciclin III as a synaptic target recognition molecule in Drosophila. Nature 374, 166-168.

Diao, F., Ironfield, H., Luan, H., Diao, F., Shropshire, W.C., Ewer, J., Marr, E., Potter, C.J., Landgraf, M., and White, B.H. (2015). Plug-and-play genetic access to drosophila cell types using exchangeable exon cassettes. Cell Rep 10, 1410-1421.

Diao, F., and White, B.H. (2012). A novel approach for directing transgene expression in Drosophila: T2A-Gal4 in-frame fusion. Genetics 190, 1139-1144.

Fox, A.N., and Zinn, K. (2005). The heparan sulfate proteoglycan syndecan is an in vivo ligand for the Drosophila LAR receptor tyrosine phosphatase. Curr Biol 15, 1701-1711. Frasch, M., Hoey, T., Rushlow, C., Doyle, H., and Levine, M. (1987). Characterization and localization of the even-skipped protein of Drosophila. EMBO J 6, 749-759.

Guan, B., Hartmann, B., Kho, Y.H., Gorczyca, M., and Budnik, V. (1996). The Drosophila tumor suppressor gene, dlg, is involved in structural plasticity at a glutamatergic synapse. Curr Biol 6, 695-706.

Hartenstein, V., Rudloff, E., and Campos-Ortega, J.A. (1987). The pattern of proliferation of the neuroblasts in the wild-type embryo of Drosophila melanogaster. Roux Arch Dev Biol 196, 473-485.

Hoang, B., and Chiba, A. (2001). Single-cell analysis of Drosophila larval neuromuscular synapses. Dev Biol 229, 55-70.

Inaki, M., Yoshikawa, S., Thomas, J.B., Aburatani, H., and Nose, A. (2007). Wnt4 is a local repulsive cue that determines synaptic target specificity. Curr Biol 17, 1574-1579.

Keshishian, H., Broadie, K., Chiba, A., and Bate, M. (1996). The drosophila neuromuscular junction: a model system for studying synaptic development and function. Annu Rev Neurosci 19, 545-575.

Kinold, J.C., Pfarr, C., and Aberle, H. (2018). Sidestep-induced neuromuscular miswiring causes severe locomotion defects in Drosophila larvae. Development 145.

Kraut, R., Menon, K., and Zinn, K. (2001). A gain-of-function screen for genes controlling motor axon guidance and synaptogenesis in Drosophila. Curr Biol 11, 417-430.

Kurusu, M., Cording, A., Taniguchi, M., Menon, K., Suzuki, E., and Zinn, K. (2008). A screen of cell-surface molecules identifies leucine-rich repeat proteins as key mediators of synaptic target selection. Neuron 59, 972-985.

Landgraf, M., Bossing, T., Technau, G.M., and Bate, M. (1997). The origin, location, and projections of the embryonic abdominal motorneurons of Drosophila. J Neurosci 17, 96429655.

Lee, H.K., Cording, A., Vielmetter, J., and Zinn, K. (2013). Interactions between a receptor tyrosine phosphatase and a cell surface ligand regulate axon guidance and glial-neuronal communication. Neuron 78, 813-826.

Lee, H.K., Wright, A.P., and Zinn, K. (2009). Live dissection of Drosophila embryos: streamlined methods for screening mutant collections by antibody staining. J Vis Exp. Menon, K.P., Carrillo, R.A., and Zinn, K. (2013). Development and plasticity of the Drosophila larval neuromuscular junction. Wiley Interdiscip Rev Dev Biol 2, 647-670. Menon, K.P., Carrillo, R.A., and Zinn, K. (2015). The translational regulator Cup controls NMJ presynaptic terminal morphology. Mol Cell Neurosci 67, 126-136. 
Nagarkar-Jaiswal, S., DeLuca, S.Z., Lee, P.T., Lin, W.W., Pan, H., Zuo, Z., Lv, J., Spradling, A.C., and Bellen, H.J. (2015a). A genetic toolkit for tagging intronic MiMIC containing genes. Elife 4.

Nagarkar-Jaiswal, S., Lee, P.T., Campbell, M.E., Chen, K., Anguiano-Zarate, S., Gutierrez, M.C., Busby, T., Lin, W.W., He, Y., Schulze, K.L., et al. (2015b). A library of MiMICs allows tagging of genes and reversible, spatial and temporal knockdown of proteins in Drosophila. Elife 4. Nose, A. (2012). Generation of neuromuscular specificity in Drosophila: novel mechanisms revealed by new technologies. Front Mol Neurosci 5, 62.

Ozkan, E., Carrillo, R.A., Eastman, C.L., Weiszmann, R., Waghray, D., Johnson, K.G., Zinn, K., Celniker, S.E., and Garcia, K.C. (2013). An extracellular interactome of immunoglobulin and LRR proteins reveals receptor-ligand networks. Cell 154, 228-239.

Patel, N.H., Schafer, B., Goodman, C.S., and Holmgren, R. (1989). The role of segment polarity genes during Drosophila neurogenesis. Genes Dev 3, 890-904.

Pipes, G.C., Lin, Q., Riley, S.E., and Goodman, C.S. (2001). The Beat generation: a multigene family encoding IgSF proteins related to the Beat axon guidance molecule in Drosophila. Development 128, 4545-4552.

Rowe, J.B. (2010). Connectivity Analysis is Essential to Understand Neurological Disorders. Front Syst Neurosci 4.

Sanchez-Soriano, N., Tear, G., Whitington, P., and Prokop, A. (2007). Drosophila as a genetic and cellular model for studies on axonal growth. Neural Dev 2, 9.

Schmid, A., Chiba, A., and Doe, C.Q. (1999). Clonal analysis of Drosophila embryonic neuroblasts: neural cell types, axon projections and muscle targets. Development 126, 4653-4689.

Siebert, M., Banovic, D., Goellner, B., and Aberle, H. (2009). Drosophila motor axons recognize and follow a Sidestep-labeled substrate pathway to reach their target fields. Genes Dev 23, 1052-1062.

Sink, H., Rehm, E.J., Richstone, L., Bulls, Y.M., and Goodman, C.S. (2001). sidestep encodes a target-derived attractant essential for motor axon guidance in Drosophila. Cell 105, 57-67. Sink, H., and Whitington, P.M. (1991). Location and connectivity of abdominal motoneurons in the embryo and larva of Drosophila melanogaster. J Neurobiol 22, 298-311.

Tan, L., Zhang, K.X., Pecot, M.Y., Nagarkar-Jaiswal, S., Lee, P.T., Takemura, S.Y., McEwen, J.M., Nern, A., Xu, S., Tadros, W., et al. (2015). Ig Superfamily Ligand and Receptor Pairs Expressed in Synaptic Partners in Drosophila. Cell 163, 1756-1769.

Taniguchi, H., Shishido, E., Takeichi, M., and Nose, A. (2000). Functional dissection of drosophila capricious: its novel roles in neuronal pathfinding and selective synapse formation. J Neurobiol 42, 104-116.

Valakh, V., Naylor, S.A., Berns, D.S., and DiAntonio, A. (2012). A large-scale RNAi screen identifies functional classes of genes shaping synaptic development and maintenance. Dev Biol 366, 163-171.

Venken, K.J., Schulze, K.L., Haelterman, N.A., Pan, H., He, Y., Evans-Holm, M., Carlson, J.W., Levis, R.W., Spradling, A.C., Hoskins, R.A., et al. (2011). MiMIC: a highly versatile transposon insertion resource for engineering Drosophila melanogaster genes. Nat Methods 8, 737-743. Winberg, M.L., Mitchell, K.J., and Goodman, C.S. (1998). Genetic analysis of the mechanisms controlling target selection: complementary and combinatorial functions of netrins, semaphorins, and IgCAMs. Cell 93, 581-591. 


\section{FIGURE LEGENDS}

Figure 1. DIP- $\alpha$ is expressed in a subset of neurons that includes MNISN-1s/RP2. (A-B) DIP- $\alpha$-T2A-GAL4 driving EGFP expression in (A) embryonic and (B) third instar larval ventral nerve cords labeled with anti-GFP (green) and anti-Eve (red). Arrowheads denote segmentally repeating Eve expressing neurons, including RP2/MNISN-1s.

(C) Dorsal larval body wall hemisegment labeled with anti-GFP (green), anti-HRP (red) and phalloidin (blue). DIP- $\alpha$ is only expressed in 1 s neurons (arrow in $\mathrm{C}$ ) and not $1 \mathrm{~b}$ neurons (arrowhead). Muscles are labeled as $\mathrm{m} 1-4$ and $\mathrm{m} 20$ in $\mathrm{C}^{\prime}$.

(D) $D I P-\alpha$-EGFP-DIP- $\alpha$ protein trap reveals DIP- $\alpha$ localization to $1 \mathrm{~s}$ (arrow) NMJ and not $1 \mathrm{~b}$ boutons (arrowhead), labeled with anti-GFP (green) and anti-HRP (red). Calibration bar is $16 \mu \mathrm{m}$ in $\mathrm{A}, 13 \mu \mathrm{m}$ in $\mathrm{B}, 50 \mu \mathrm{m}$ in $\mathrm{C}$, and $4 \mu \mathrm{m}$ in D. See also Figure S1.

Figure 2. DIP- $\alpha$ is required for MNISN-1s branch formation on muscle 4.

(A-C) Larval neuromuscular junctions labeled with anti-GFP (green) and anti-HRP (red) from (A) heterozygous DIP- $\alpha$-GAL4 mutants, (B) homozygous DIP- $\alpha$-GAL4 mutants and (C) rescue of homozygous DIP- $\alpha$-GAL4 mutants by expressing a UAS-DIP- $\alpha$ construct in DIP- $\alpha$ expressing cells, including MNISN-1s. DIP- $\alpha$ is only expressed in 1s neurons (green) (arrow in A) and not $1 \mathrm{~b}$ neurons (arrowhead).

(D-F) Frequency of $1 \mathrm{~s}$ innervation of muscle 4 from (D) mutants and rescue, (E) overexpression of UAS-DIP- $\alpha$ or (F) UAS-DIP- $\alpha$-RNAi. n (animals $/$ hemisegments) $=$ (D) (12/60), (10/30), (18/100), (18/96), (9/56), (14/69), (E) (8/48), (6/30), (9/54), (F) (6/30), (12/71), (10/60), (10/59) (respectively). Calibration bar is $9 \mu \mathrm{m}$ in A-C. ${ }^{* *} \mathrm{p}<0.001$, $* * * \mathrm{p}<0.0001$. Error bars represent SEM.

Figure 3. MNISN-1s axons in DIP- $\alpha$ mutants produce protrusions on $\mathrm{m} 4$ but are unable to form stable $1 \mathrm{~s}$ branches.

(A-D) Embryonic NMJs labeled with anti-GFP (green), anti-HRP (red) and phalloidin (blue) from $(\mathrm{A}, \mathrm{C})$ heterozygous and $(\mathrm{B}, \mathrm{D})$ homozygous DIP- $\alpha-G A L 4$ mutants. $(\mathrm{A}-\mathrm{B})$ are st15 embryos; (C-D) are st17 embryos. MNISN-1s protrusions over $\mathrm{m} 4$ form late in embryonic development (C) while protrusions over $\mathrm{m} 2$ form earlier (A). Note that MNISN-1s axon protrusions form properly over dorsal muscles, including $\mathrm{m} 2$, at st15 in (A) controls and (B) DIP- $\alpha$ mutants and over $\mathrm{m} 4$ at st17 in controls (C) and DIP- $\alpha$ mutants (D). Also, $\wedge$ indicates all filopodia in (A) and (B), and m4 filopodia in (C) and (D). * indicates st17 nonm4 filopodia.

(E-F) First instar larva labeled with anti-GFP (green), anti-HRP (red) and phalloidin (blue) from $D I P-\alpha-G A L 4$ heterozygotes and (F) DIP- $\alpha-G A L 4$ homozygous mutants. Note $1 \mathrm{~b}$ boutons present in both $1^{\text {st }}$ instar larva (arrowhead in E,F), but only heterozygote has $1 \mathrm{~s}$ boutons (arrow in E). Calibration bar is $8 \mu \mathrm{m}$ in $\mathrm{A}, \mathrm{B}, 4 \mu \mathrm{m}$ in $\mathrm{C}, \mathrm{D}$ and $6 \mu \mathrm{m}$ in $\mathrm{E}, \mathrm{F}$.

Figure 4. Loss of DIP- $\alpha$ causes selective loss of MNISN-1s branches on proximal muscles and ectopic innervation of distal muscles.

$(A, B)$ Dorsal body wall hemisegments labeled with anti-GFP (green), anti-HRP (red) and phalloidin (blue) from (A) heterozygous DIP- $\alpha$-GAL4 animals, and (B) homozygous DIP- $\alpha$ GAL4 mutants. Arrow denotes 1s boutons in (A), arrowhead denotes $1 \mathrm{~b}$ boutons, and * 
denote muscles in mutant that have lost 1 s innervation. Overgrown 1 s arbors on $\mathrm{m} 2$ and $\mathrm{m} 1$ are circled in mutants.

(C) Quantification of the frequency of innervation of MNISN-1s neurons on the respective muscles from the above genotypes. $n$ (animals $/$ hemisegments $)=(16 / 30),(12 / 30)$ (respectively). Calibration bar is $60 \mu \mathrm{m}$.

Figure 5. Dpr10 is expressed in muscles and can localize to the SSR and bind to DIP- $\alpha$. (A,C) Embryonic ventral nerve cord from (A) dpr6-GAL4 and (C) dpr10-GAL4 driving EGFP expression, labeled with anti-GFP (green), anti-Eve (red) and anti-Elav (blue). dpr6 is expressed in aCC and RP2 (MNISN-1s) neurons, while $d p r 10$ is not expressed in these Eve+ cells (see also Figure S4).

(B,D) Larval dorsal body wall dorsal hemisegments from (B) dpr6-GAL4 or (D) dpr10-GAL4 driving GFP expression, labeled with anti-GFP (green), and anti-HRP (red). Muscles are labeled m4-m1, m20. Dpr10 is also expressed in unknown neurons ( ${ }^{\wedge}$ in 4D denotes axon). (E) Larval m4 NMJ from animals expressing Dpr10-V5 using MHC-GeneSwitch-GAL4 in the absence of RU486 (low-level (leaky) muscle expression only), labeled with anti-V5 (green), anti-HRP (red) and anti-Dlg (blue). 1b and 1s neurons are marked with arrowhead and arrow, respectively.

$(\mathrm{F}, \mathrm{G})$ Larval m4 NMJ from either (G) control $w^{1118}$ or $(\mathrm{H}) 24 B-G A L 4>\mathrm{UAS}-\mathrm{Dpr} 10$-V5 (high level pan-muscle expression). Live tissue was incubated with DIP- $\alpha$-AP 5 protein (see Methods) and then labeled with anti-AP (green) and anti-HRP (red). Calibration bar is $15 \mu \mathrm{m}$ in $\mathrm{A}, \mathrm{C}, 60 \mu \mathrm{m}$ in $\mathrm{B}, \mathrm{D}, 12 \mu \mathrm{m}$ in $\mathrm{E}$, and $18 \mu \mathrm{m}$ in $\mathrm{F}, \mathrm{G}$.

Figure 6. Dpr10 is required for MNISN-1s innervation of muscle 4.

(A-C) Larval m4 NMJs labeled with anti-DLG (green) and anti-HRP (red) from (A) control $w^{1118}$ animals, (B) $d p r 10^{14-5} / D f$ null mutants and (C) dpr6-GAL4/Df. The difference in antiDlg staining intensity allows us to differentiate $1 \mathrm{~b}$ and $1 \mathrm{~s}$ NMJs. $1 \mathrm{~b}$ and 1 s neurons are marked with arrowhead and arrow, respectively.

(D-F) Frequency of 1s innervation of m4 from (D) dpr6 and dpr10 mutants and (E) overexpression of UAS-Dpr10-V5 and (F) UAS-dpr10-RNAi. $\mathrm{n}$ (animals $/$ hemisegments) = (D) (8/62), (12/70), (11/60) and (E) (8/48), (6/36), (5/30), (8/47), (F) (8/48), (8/48), (10/60), (8/48) (respectively). Calibration bar is $16 \mu \mathrm{m}$ in A-C. Error bars represent SEM. $* * * \mathrm{p}<0.0001$.

Figure 7. Temporal and spatial expression of $d p r 10$ in embryonic muscles.

(A,C,E) Embryonic dpr10>EGFP abdominal body wall hemisegments labeled with anti-GFP (green), anti-Fas2 (red) and phalloidin (blue) from (A) late st14/early st15, (C) late st15/early st16, and (E) late st16/early st17. Open arrowhead denotes Intersegmental Nerve (ISN), which carries dorsal neurons.

$(B, D, F)$ Cartoon representation of an embryonic hemisegment. Green muscles are those that express Dpr10 at the respective developmental stage while blue muscles do not express Dpr10.

(G) Heat map quantification of the frequency of $d p r 10>E G F P$ expression in each muscle at each developmental stage, where 1 represents $100 \%$ probability of expression. $n$ (embryos) $=7,6,8$ (respectively). Calibration bar is $19 \mu \mathrm{m}$. 
Figure S1. DIP- $\alpha$ is expressed in a subset of VNC neurons and DIP- $\alpha$ protein is enriched in the neuropil.

(A) Ventral confocal projections of VNC in st16 embryos. Arrow denotes MNSNb/d-1s.

(B) Dorsal confocal projections of VNC in larval animals depicting GFP expression of DIP- $\alpha$ GAL4. labeled with anti-GFP (green) and anti-HRP (red). Arrow denotes MNISN-1s.

(C) Ventral larval body wall muscles labeled with anti-GFP (green), anti-HRP (red), and phalloidin (blue), showing that DIP- $\alpha-G A L 4$ is expressed in MNSNb/d-1s motor neurons as well.

(D) Ventral nerve cord from DIP- $\alpha$-EGFP-DIP- $\alpha$ protein trap larva, labeled with anti-GFP (green) and anti-HRP (red), showing that DIP- $\alpha$ also localizes to the 1 s dendritic processes. Calibration bar is $19 \mu \mathrm{m}$ in $\mathrm{A}, 17 \mu \mathrm{m}$ in $\mathrm{B}, 40 \mu \mathrm{m}$ in $\mathrm{C}$, and $18 \mu \mathrm{m}$ in D.

Figure S2. DIP- $\alpha$ protein from a transgene localizes normally in rescue animals. $(A, B)$ The DIP- $\alpha$ rescue construct, tagged with a c-terminal Myc tag, labeled with (A) antiMyc (green), anti-HRP (red) and anti-Dlg (blue) or (B) anti-DIP- $\alpha$ (green), anti-HRP (red) or phalloidin (blue). Note localization only to 1s boutons (arrows) and not $1 \mathrm{~b}$ boutons (arrowheads). Open arrowheads denote the ISN. Calibration bar is $10.5 \mu \mathrm{m}$.

Figure S3. DIP- $\alpha$ is expressed in MNISN-1s motor neurons in st14 embryos. (A) An embryonic ventral nerve cord labeled with anti-GFP (green) and anti-Fas2 (red) from a DIP- $\alpha$-GAL4 driving GFP in st14 embryos. Calibration bar is $14 \mu \mathrm{m}$.

Figure S4. Dpr10 is expressed is in a subset of neurons in the embryonic ventral nerve cord.

(A) An embryonic ventral nerve cord from $d p r 10>$ EGFP labeled with anti-GFP (green) and anti-Elav (red) from sections below the MNISN-1s neuron cell bodies seen in Figure 4. Calibration bar is $11 \mu \mathrm{m}$. 

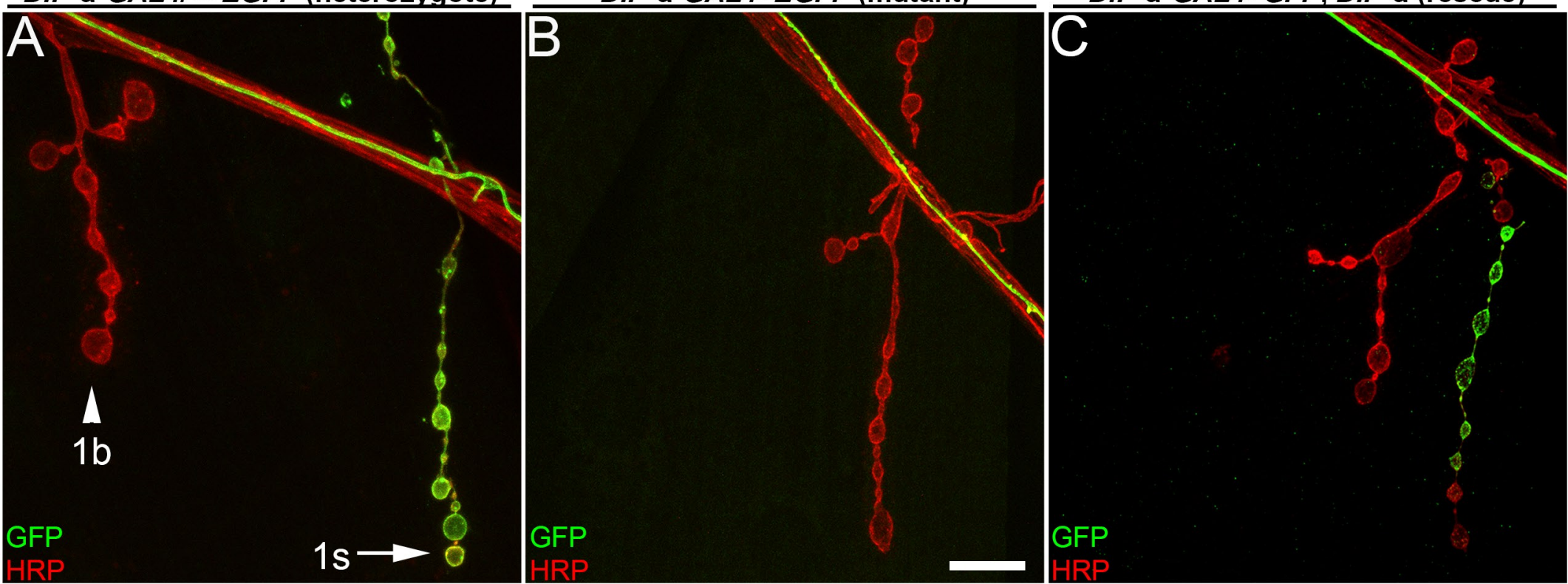

$D$

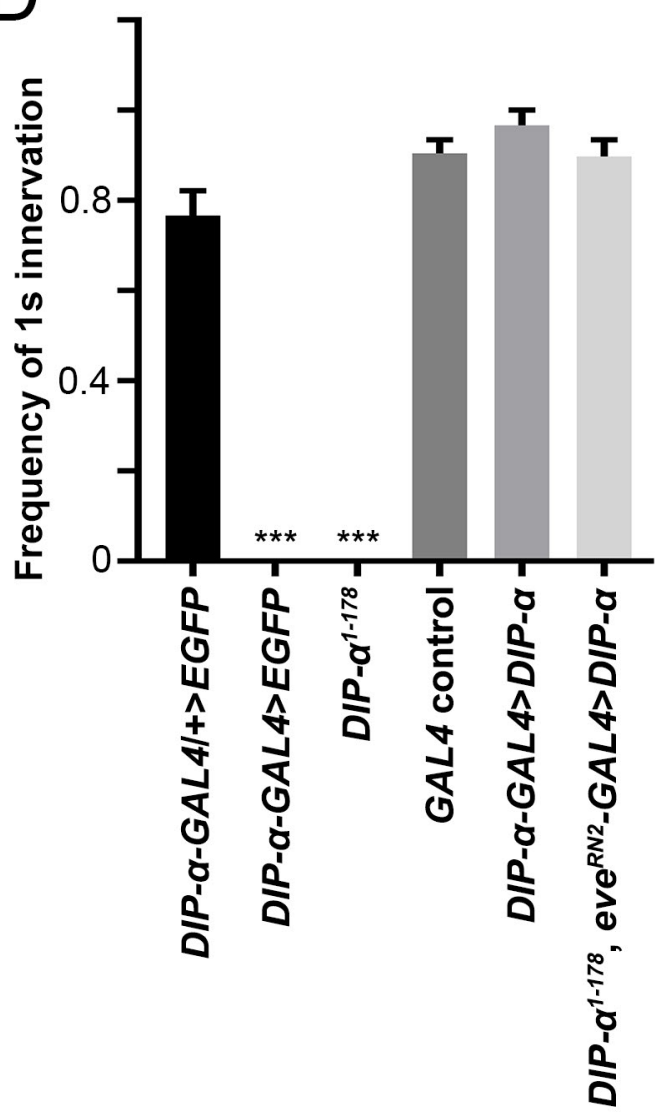

E

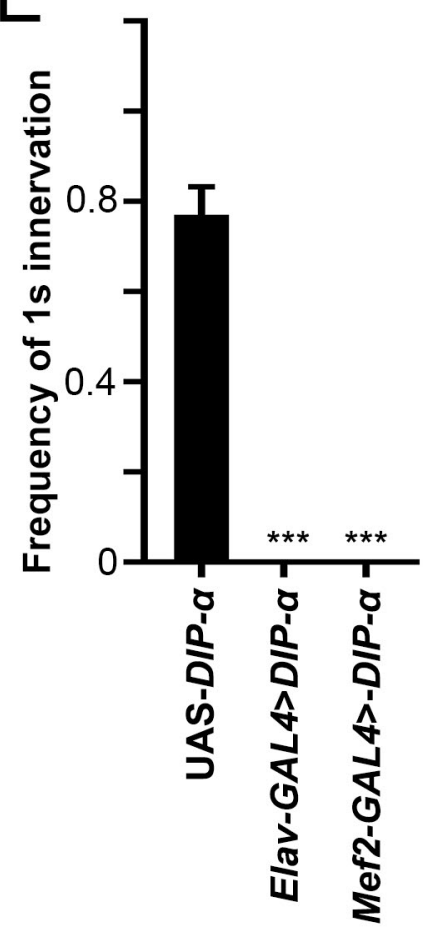

$E$

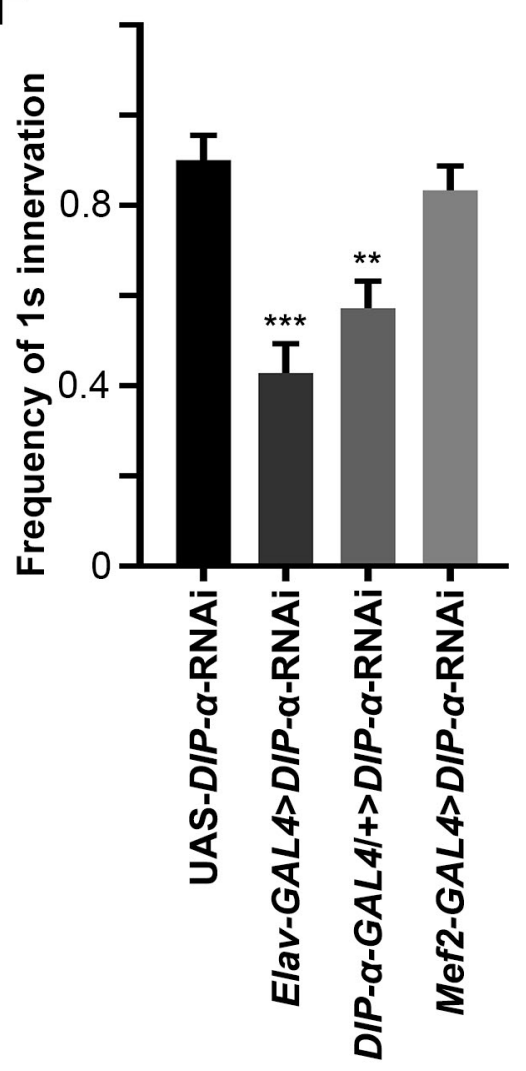

Figure 2 


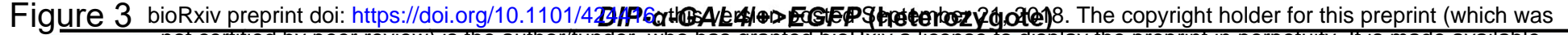

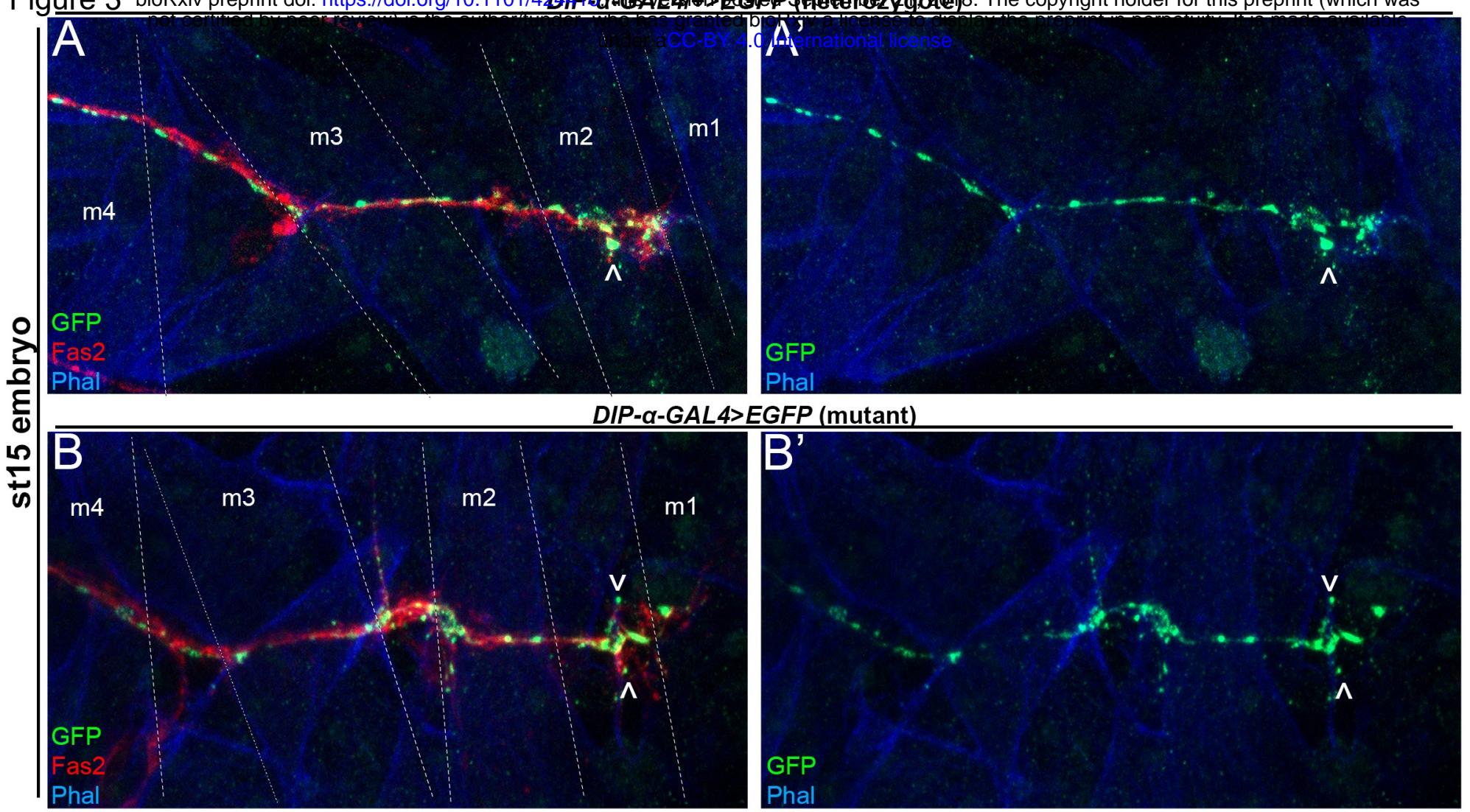

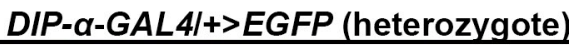
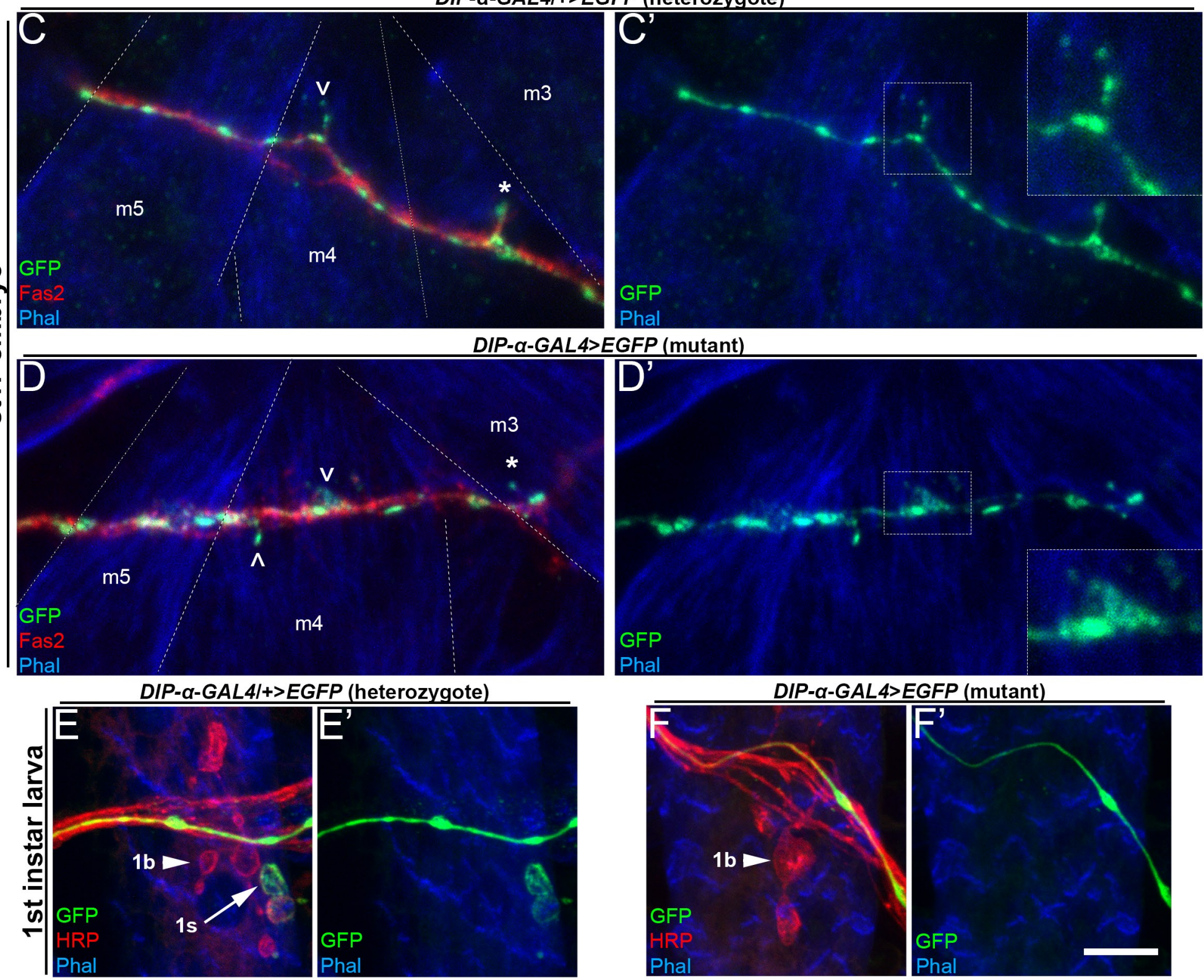


\section{Figure 5}

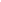



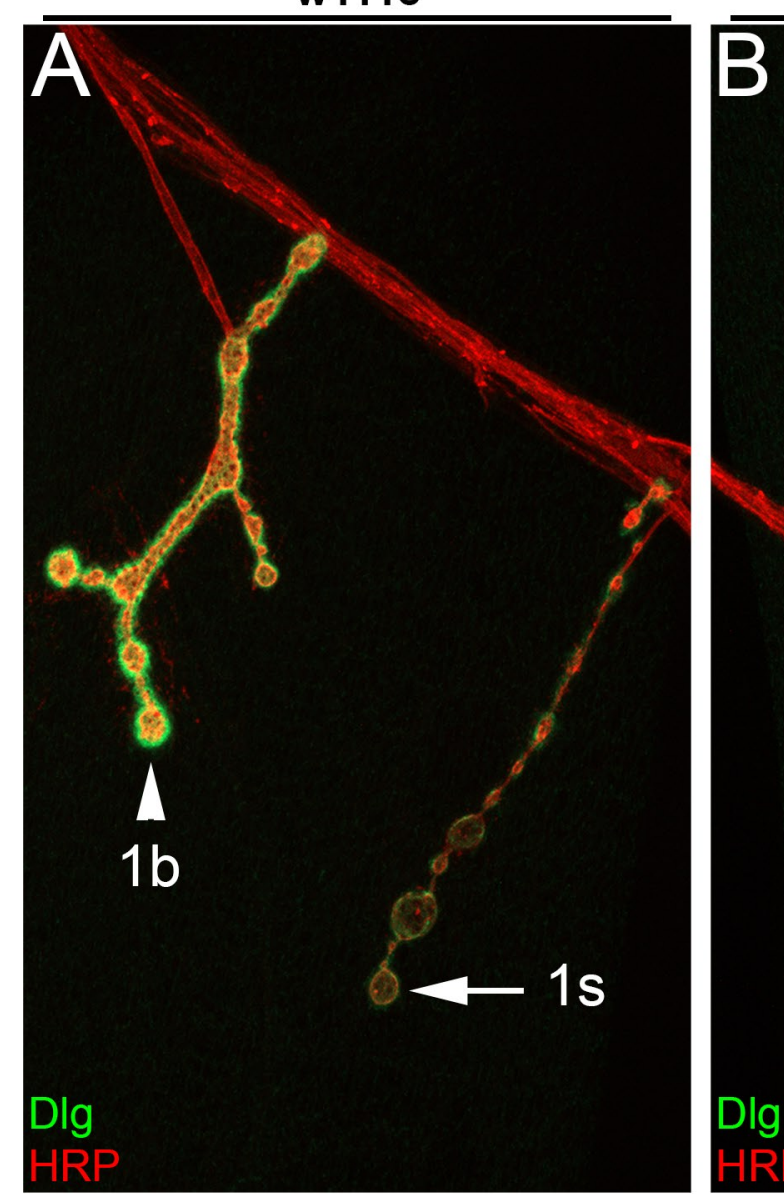

D
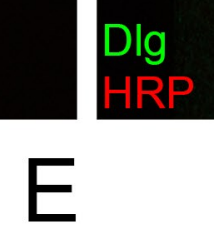

Dlg
HRP

F
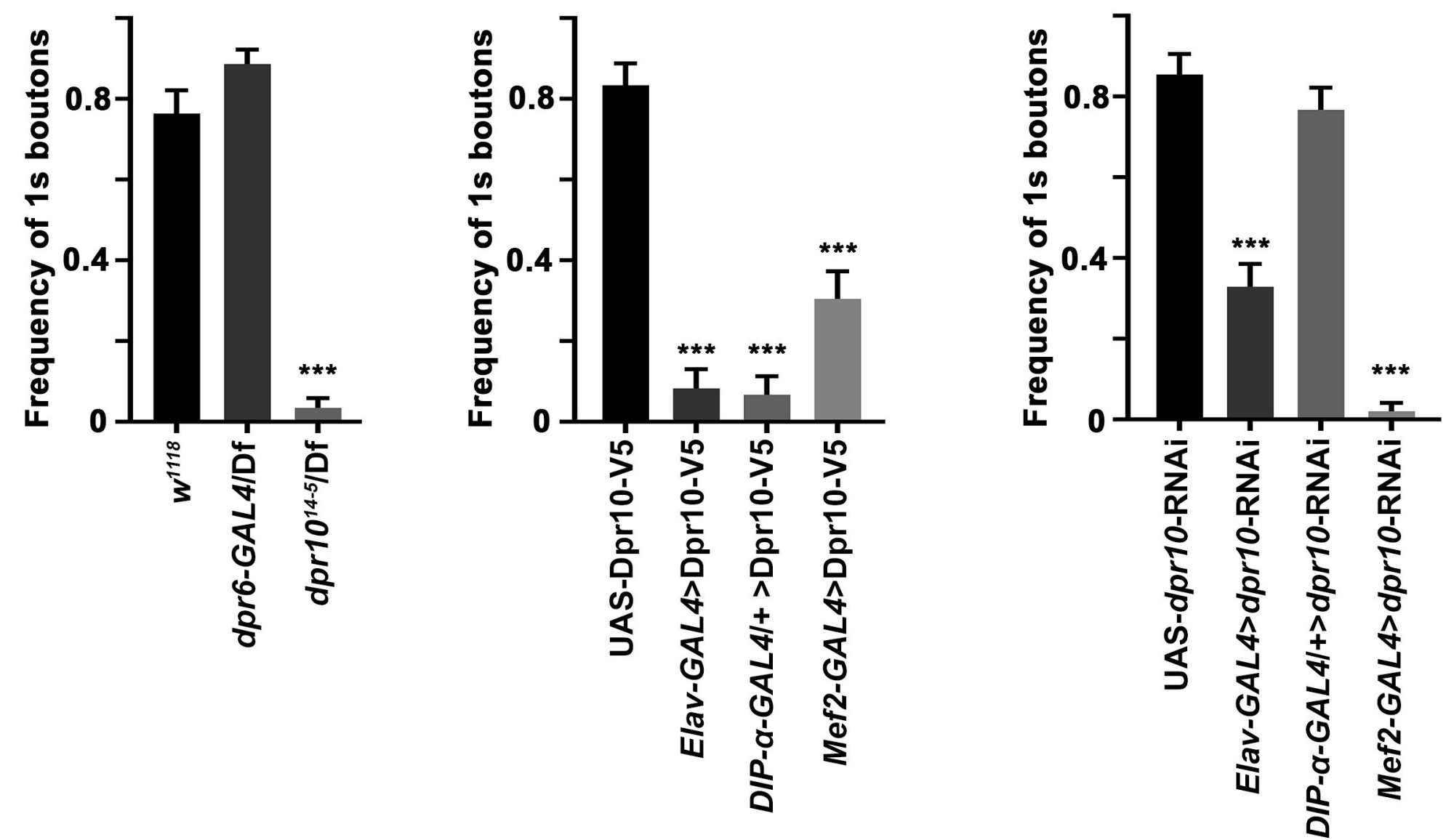
Figure 7

dpr10-GAL4>EGFP

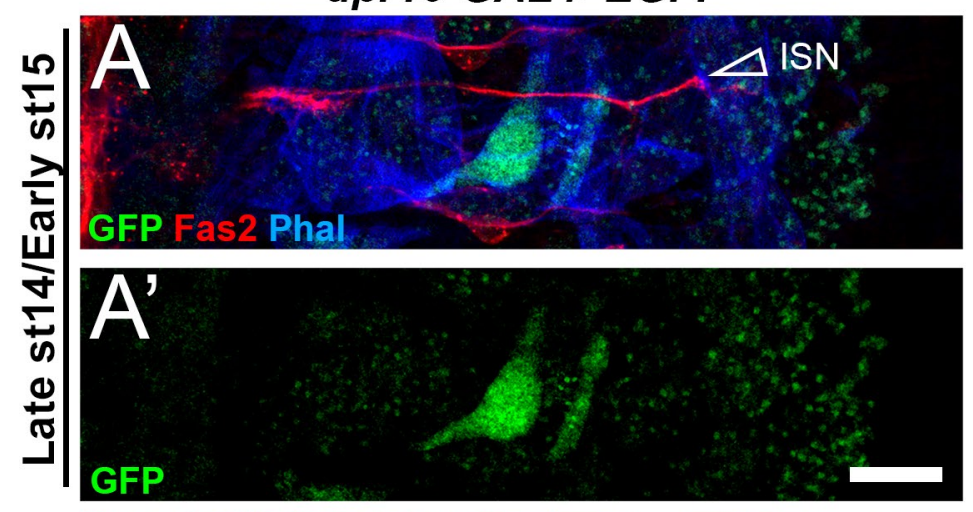

B
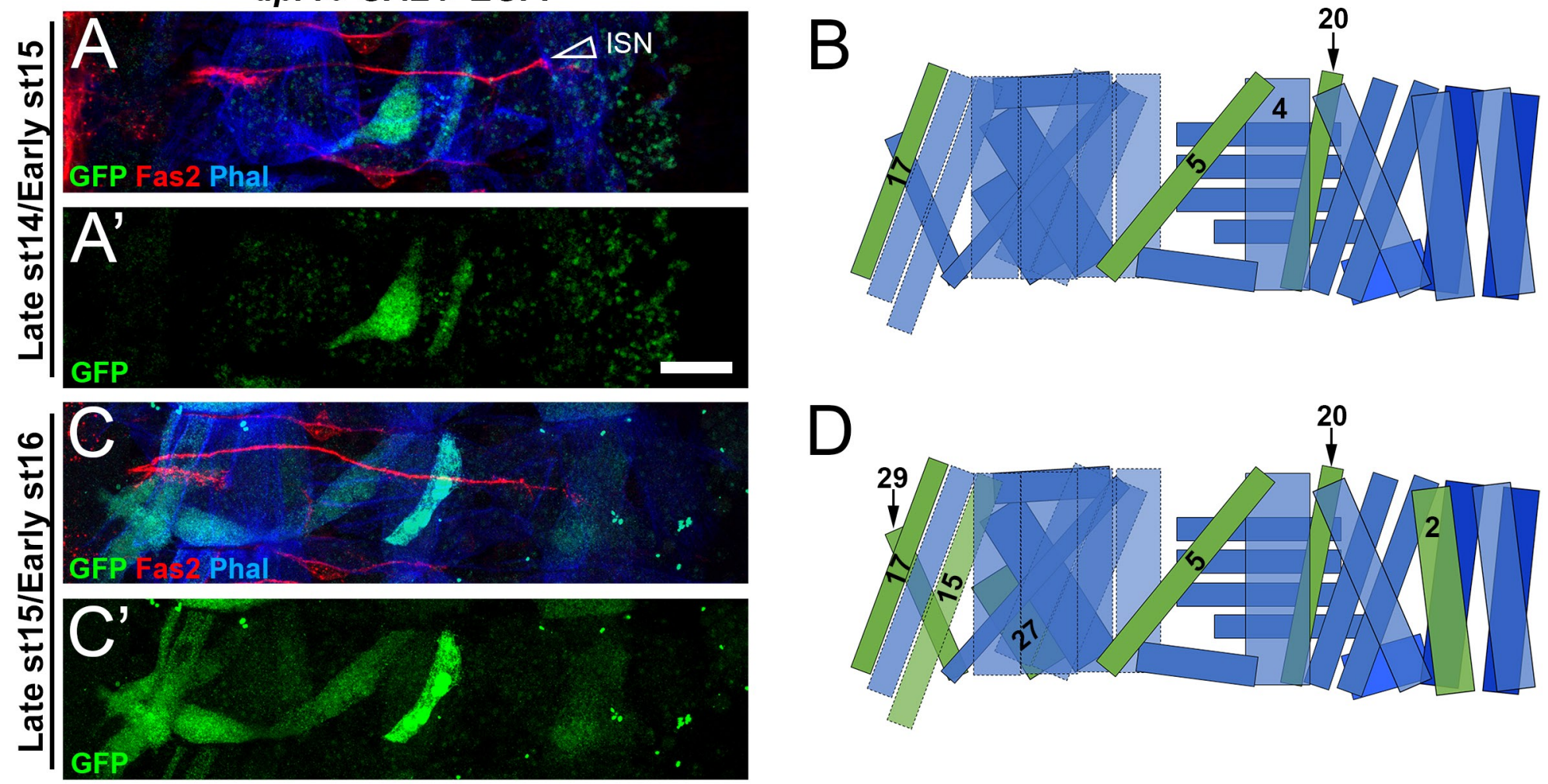

D
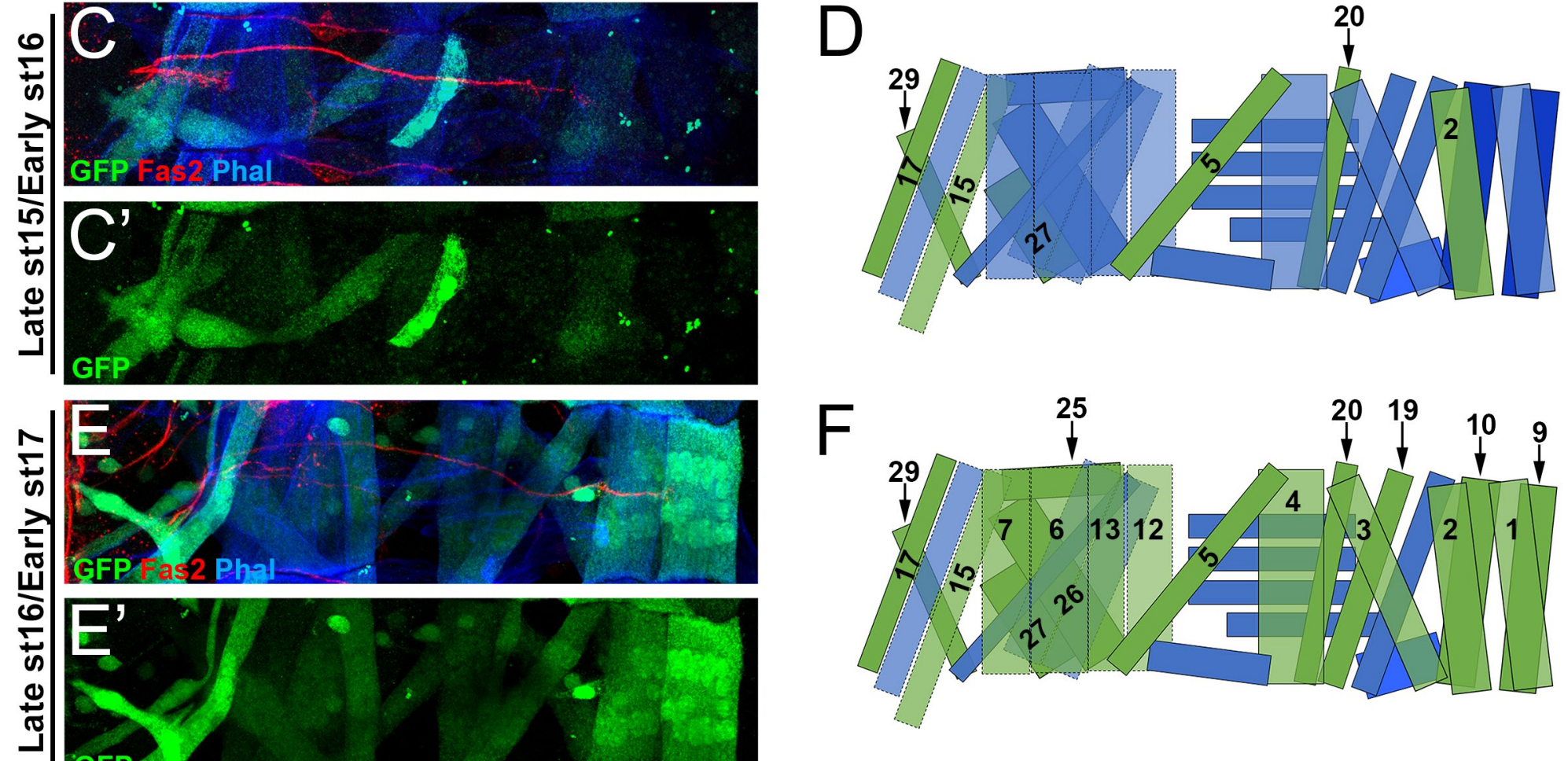

E)

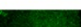

F

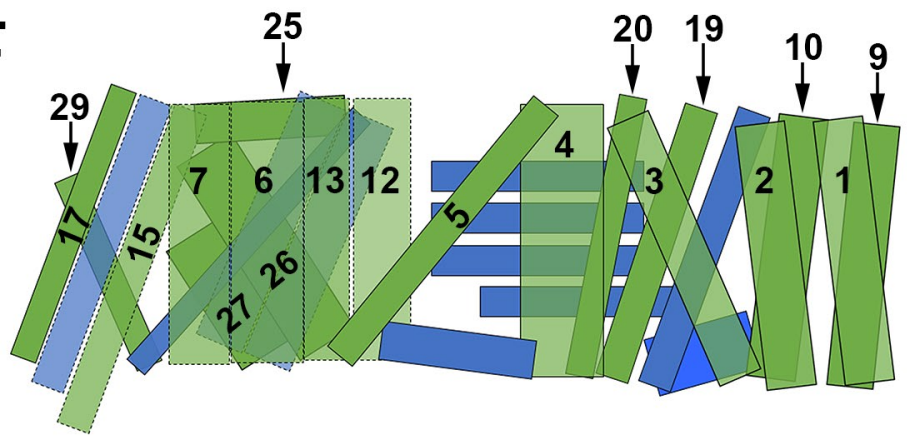

GFP

$G$

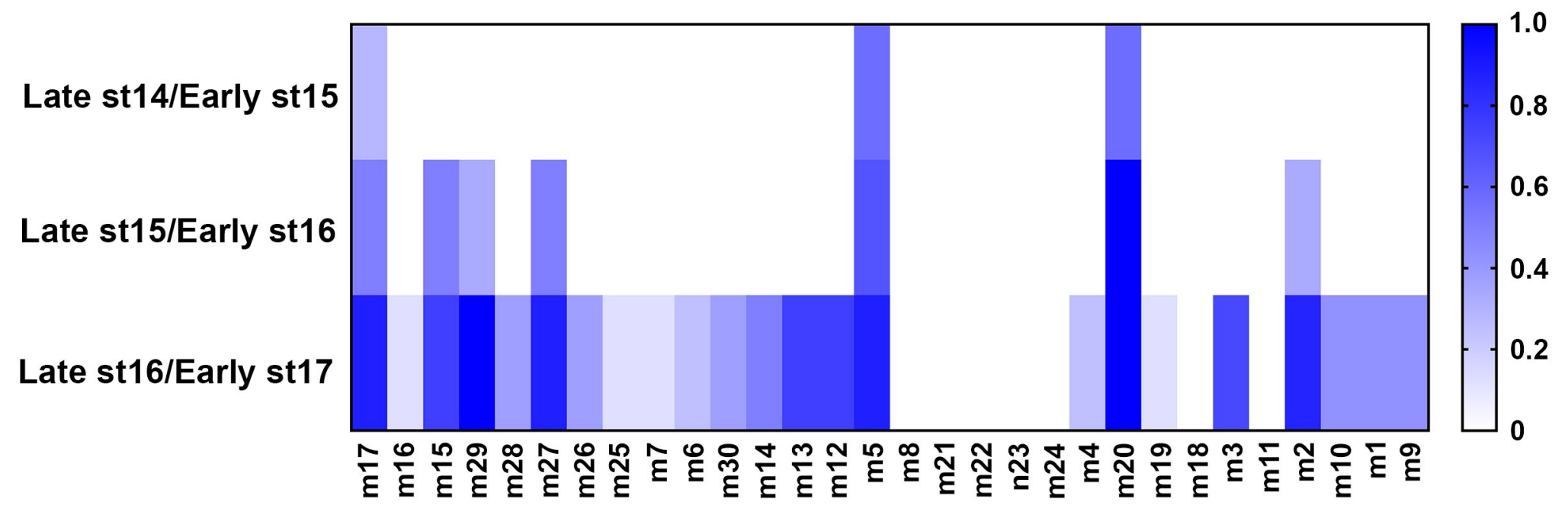


Figure S1 DIP-a-GAL4I+>EGFP

$D|P-\alpha-G A L 4|+>E G F P$
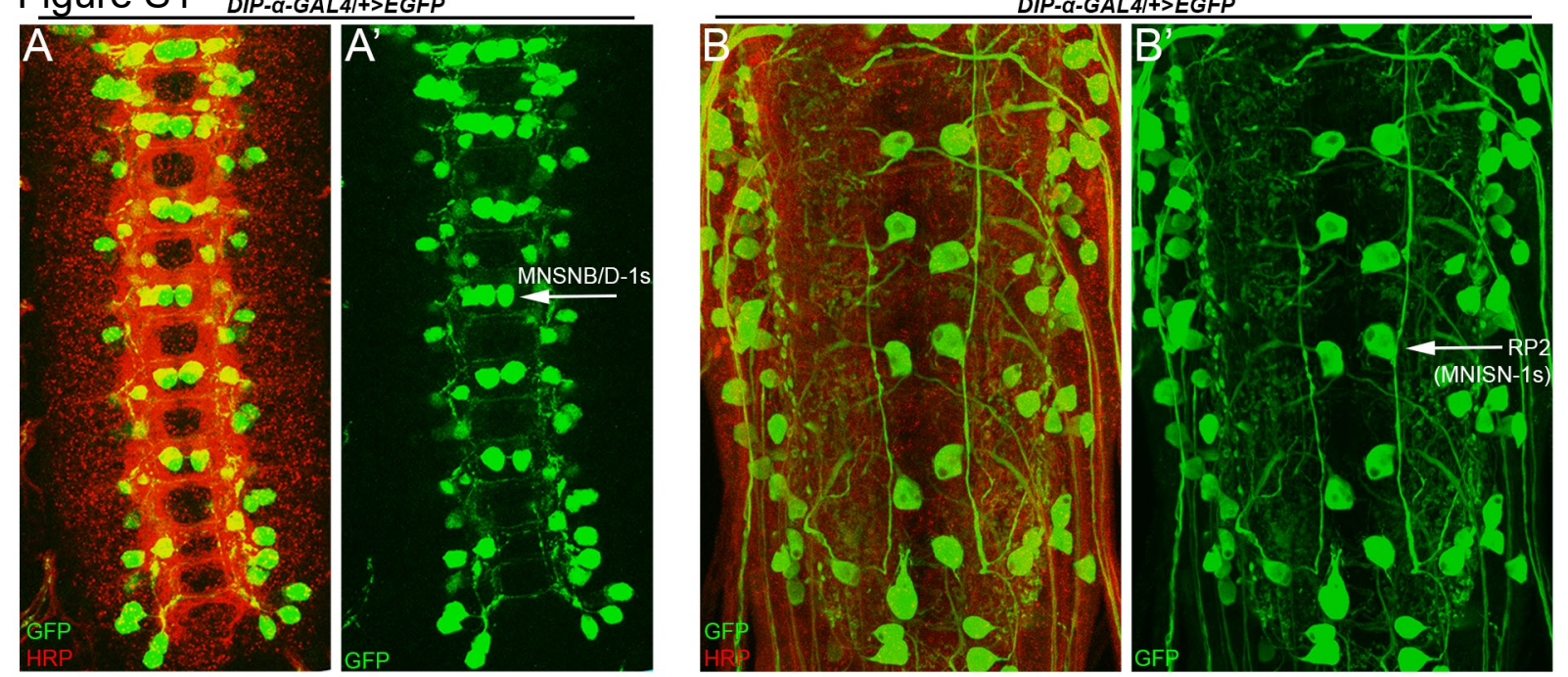

$\bar{C}$

DIP- $\alpha-G A L 4 \mid+>E G F P$

D

DIP-a-EGFP-DIP- $a$

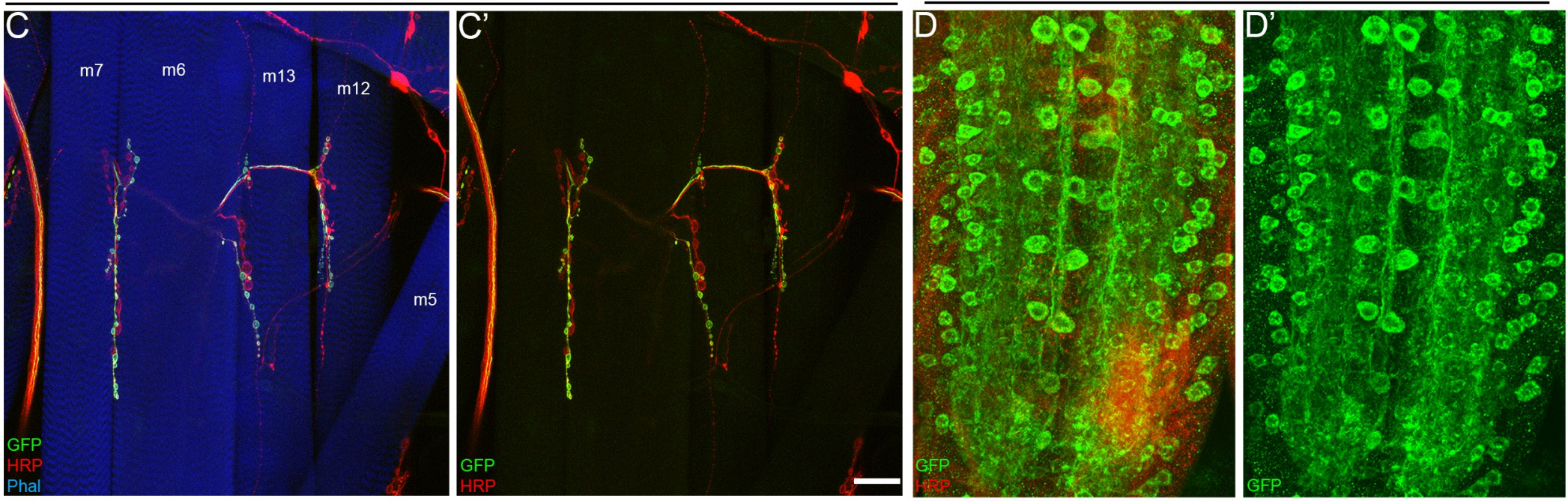




$$
\text { A }
$$

$\mathbf{A}^{\prime}$

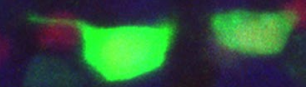

อ 